\title{
Analysis of fuzzy Hamacher aggregation functions for uncertain multiple attribute decision making
}

DOI:

10.1016/j.ins.2016.12.045

\section{Document Version}

Accepted author manuscript

Link to publication record in Manchester Research Explorer

\section{Citation for published version (APA):}

Tang, X., Fu, C., Xu, L., \& Yang, S. (2017). Analysis of fuzzy Hamacher aggregation functions for uncertain multiple attribute decision making. Information Sciences, 387. https://doi.org/10.1016/j.ins.2016.12.045

\section{Published in:}

Information Sciences

\section{Citing this paper}

Please note that where the full-text provided on Manchester Research Explorer is the Author Accepted Manuscript or Proof version this may differ from the final Published version. If citing, it is advised that you check and use the publisher's definitive version.

\section{General rights}

Copyright and moral rights for the publications made accessible in the Research Explorer are retained by the authors and/or other copyright owners and it is a condition of accessing publications that users recognise and abide by the legal requirements associated with these rights.

\section{Takedown policy}

If you believe that this document breaches copyright please refer to the University of Manchester's Takedown Procedures [http://man.ac.uk/04Y6Bo] or contact uml.scholarlycommunications@manchester.ac.uk providing relevant details, so we can investigate your claim.

\section{OPEN ACCESS}




\section{Accepted Manuscript}

Analysis of fuzzy Hamacher aggregation functions for uncertain multiple attribute decision making

Xiaoan Tang , Chao Fu, Dong-Ling Xu, Shanlin Yang

PII: S0020-0255(16)32280-0

DOI: $10.1016 / j$.ins.2016.12.045

Reference: INS 12680

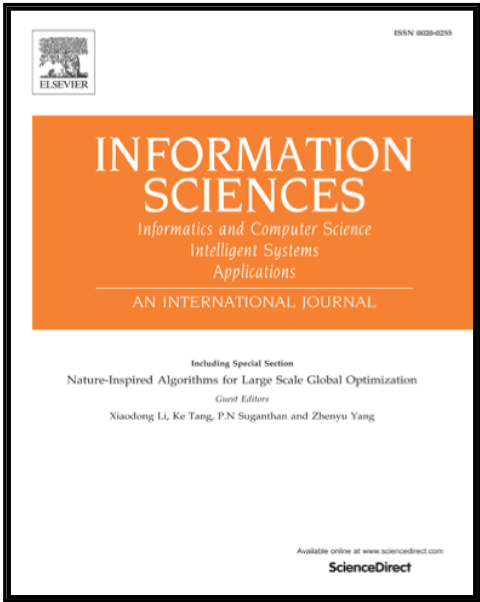

To appear in:

Information Sciences

Received date: 20 November 2015

Revised date: $\quad 25$ November 2016

Accepted date: $\quad 31$ December 2016

Please cite this article as: Xiaoan Tang, Chao Fu, Dong-Ling Xu, Shanlin Yang, Analysis of fuzzy Hamacher aggregation functions for uncertain multiple attribute decision making, Information Sciences (2017), doi: 10.1016/j.ins.2016.12.045

This is a PDF file of an unedited manuscript that has been accepted for publication. As a service to our customers we are providing this early version of the manuscript. The manuscript will undergo copyediting, typesetting, and review of the resulting proof before it is published in its final form. Please note that during the production process errors may be discovered which could affect the content, and all legal disclaimers that apply to the journal pertain. 


\section{Analysis of fuzzy Hamacher aggregation functions for uncertain multiple} attribute decision making

Xiaoan Tang ${ }^{\mathrm{a}, \mathrm{b}}$, Chao $\mathrm{Fu}^{\mathrm{a}, \mathrm{b}}$, Dong-Ling $\mathrm{Xu}^{\mathrm{c}, *}$, Shanlin Yang ${ }^{\mathrm{a}, \mathrm{b}}$

${ }^{a}$ School of Management, Hefei University of Technology, Hefei, Box 270, Hefei

230009, Anhui, P.R. China

${ }^{b}$ Key Laboratory of Process Optimization and Intelligent Decision-making, Ministry

of Education, Hefei, Box 270, Hefei 230009, Anhui, P.R. China

${ }^{c}$ Manchester Business School, The University of Manchester, Manchester M15 6PB,

$$
U K
$$

\section{Abstract}

As generalizations of algebraic and Einstein t-norms and t-conorms, Hamacher t-norm and t-conorm have been widely applied in fuzzy multiple attribute decision making (MADM) to combine assessments on each attribute, which are generally expressed by Atanassov's intuitionistic fuzzy (AIF) numbers, interval-valued intuitionistic fuzzy (IVIF) numbers, hesitant fuzzy, (HF) elements, and dual hesitant fuzzy (DHF) elements. Due to the fact that AIF numbers and HF elements are special cases of IVIF numbers and DHF elements, respectively, two propositions can be established from analyzing numerical examples and real cases concerning MADM with IVIF and DHF assessments in the literature: (1) the monotonicity of alternative scores derived from Hamacher arithmetic and geometric aggregation operators with respect to the parameter $r$ in Hamacher t-norm and t-conorm; and (2) the relationship between alternative scores generated by Hamacher arithmetic and geometric aggregation operators, given the same $r$. Here, we provide the theoretical proof of these two propositions in the context of MADM with IVIF and DHF assessments. With the theoretical support of these propositions, the meaning of $r$ in MADM is explained, and a new method is proposed to compare alternatives in MADM with consideration of all possible values of $r$. Two numerical examples are solved by the proposed method and the other two existing methods to demonstrate the applicability and validity of the proposed method and highlight its advantages.

Keywords: Hamacher t-norm and t-conorm; Interval-valued intuitionistic fuzzy set; Dual hesitant fuzzy set; Arithmetic and geometric aggregation operators; Monotonicity 


\section{Introduction}

Decision making can be considered a mental process in which human beings make a choice among several alternatives. However, with the increasing complexity of real decision problems, decision makers frequently face the challenge of characterizing their preferences in an uncertain context. This opens an important application field of fuzzy set theory and granular computing techniques: fuzzy decision making $[2,8,10,14,23,33,39,41,47]$. As stated by Pedrycz and Chen [25], fuzzy decision making including its underlying methodology, the plethora of algorithmic developments, and a rich and diversified slew of application studies form a cornerstone of fuzzy sets. More importantly, it plays a key role in fuzzy decision making to combine multiple pieces of uncertain information represented by the extensions of fuzzy set [46] such as Atanassov's intuitionistic fuzzy (AIF) set [3], interval-valued intuitionistic fuzzy (IVIF) set [4], hesitant fuzzy (HF) set [32], and dual hesitant fuzzy (DHF) set [49]. To address such combination, various aggregation functions or operators have been designed and applied in multiple attribute decision making (MADM) [5,7,13,16,19,20,22,30,34,35,37,38,43,48].

Firstly, many efforts have been made concerning the combination of AIF or IVIF assessments. Beliakov et al. [5] developed the median aggregation operators for AIF sets and interval-valued fuzzy sets. Garg [13] constructed a number of generalized intuitionistic fuzzy interactive geometric interaction operators using Einstein t-norm and t-conorm. Xia et al. [38] designed intuitionistic fuzzy weighted averaging and geometric operators based on Archimedean t-norm and t-conorm [11]. Liao and $\mathrm{Xu}$ [21] proposed a family of intuitionistic fuzzy hybrid weighted aggregation operators in which the properties of idempotency and boundedness are satisfied. As an important style of the Archimedean t-norm and t-conorm, Hamacher t-norm and t-conorm [15] were used to construct a number of intuitionistic fuzzy Hamacher aggregation operators based on the unordered and ordered weighted averaging operators (OWA) [16]. Chen et al. [9] presented the IVIF aggregation operators for group decision making. Many Hamacher aggregation operators of IVIF information were also developed using the ordered weighted geometric operator [22,39].

Secondly, the combination of HF or DHF assessments has also been investigated widely in the literature. $\mathrm{Xia}$ and $\mathrm{Xu}$ [37] proposed many aggregation operators for $\mathrm{HF}$ information based on weighted averaging and geometric operators. Liao and $\mathrm{Xu}$ [20] constructed a series of new HF hybrid arithmetic aggregation operators satisfying idempotency and keeping the advantages of HF hybrid averaging and geometric operators developed by $\mathrm{Xia}$ and $\mathrm{Xu}$ [37]. In particular, Hamacher t-norm and t-conorm were used to develop a family of HF aggregation operators [30,48]. With regard to the aggregation for DHF information, several aggregation operators and 
power aggregation operators were constructed based on Archimedean t-norm and t-conorm [34,35]. Ju et al. [19] used Hamacher t-norm and t-conorm to develop some aggregation operators for DHF information.

It is worth mentioning that Hamacher t-norm and t-conorm can reduce to algebraic and Einstein t-norms and t-conorms when the parameter $r$ in Hamacher t-norm and t-conorm is set as 1 and 2, respectively [38]. For this reason, the aggregation operators based on algebraic and Einstein t-norms and t-conorms of the above four kinds of fuzzy assessments are not reviewed individually. This also indicates why fuzzy Hamacher aggregation operators are addressed in this paper.

Existing studies concerning fuzzy Hamacher aggregation operators $[16,19,22,30,38,39,48]$ reveal that they are generally divided into arithmetic and geometric aggregation operators. It can be found from numerical examples or real cases in existing studies that there are two important rules which govern the two types of aggregation operators in the context of fuzzy MADM: (1) the scores of the decision alternatives under consideration decrease and increase with the increase of the parameter $r$ in the Hamacher t-norm and t-conorm when the arithmetic and geometric aggregation operators are applied, respectively; and (2) the scores of the decision alternatives generated by the arithmetic aggregation operator are always larger than those generated by the geometric aggregation operator, regardless of what the parameter $r$ is equal to. To the best of our knowledge, existing studies have only shown the results of calculations in numerical examples or case studies which indicate these two rules, but have not provided a theoretical analysis of such results. Although a small number of researchers (e.g., [20,21]) have discussed the relationship between arithmetic and geometric averaging operators in the AIF or HF environment, it cannot be directly extended to the situation of the arithmetic and geometric averaging operators developed based on Hamacher t-norm and t-conorm. The above analysis shows that it is necessary to theoretically discuss the relationship between arithmetic and geometric averaging operators developed based on Hamacher t-norm and $\mathrm{t}$-conorm in various fuzzy contexts. This is the first motivation of this paper.

Except the above, previous studies [16,19,22,26,30,39,48] of Hamacher aggregation operators in the context of fuzzy MADM have shown the influence of the parameter $r$ in Hamacher t-norm and t-conorm on decision results by a sensitivity analysis of $r$ only. However, two key points have been omitted in these studies: (1) what is the meaning of the parameter $r$; and (2) how is this parameter determined in MADM. The meaning of the parameter $r$ in these studies is typically unclear, and its determination is generally arbitrary and subjective $[19,22,48]$, which may negatively influence the rationality of decision results. To guarantee the rationality of decisions made with consideration of the parameter $r$, the two key points about $r$ in MADM 
need to be addressed, which forms the second motivation of this paper. As a whole, it is necessary and important to analyze the two above-mentioned rules of Hamacher arithmetic and geometric averaging operators in various fuzzy contexts from a theoretical point of view, and to address the two key points concerning the parameter $r$ in MADM.

In this paper, following the above motivations, we first present two propositions to cover the two rules, and then prove them theoretically when handling MADM problems with IVIF or DHF assessments. The situations of MADM with AIF or HF assessments are covered because IVIF and DHF assessments can reduce to AIF and HF assessments, respectively. Based on the two propositions, we associate the meaning of the parameter $r$ in Hamacher t-norm and t-conorm with the risk attitude of a decision maker and give relevant explanations. Specifically, to avoid the negative influence of arbitrary or subjective $r$ values on decision results in MADM, a new method to compare alternatives is proposed by using the mean scores of alternatives with consideration of all possible values of $r$.

In short, the main contributions of this paper include the following: (1) the construction of two propositions concerning the two types of Hamacher aggregation operators in the context of fuzzy MADM; (2) the theoretical proof of the two propositions in MADM with IVIF and DHF assessments; (3) the analysis of the meaning of the parameter $r$ in Hamacher aggregation operators; and (4) the development of a new method for ranking alternatives in MADM problems with DHF assessments, by following the two propositions.

The rest of this paper is organized as follows. The necessary preliminaries are briefly reviewed in Section 2. Section 3 conducts an analysis of MADM with IVIF and DHF assessments in the literature, before presenting propositions concerning the two rules found. In Section 4, these propositions are proven theoretically in the context of MADM with IVIF and DHF assessments. In Section 5, the meaning of the parameter $r$ in the Hamacher t-norm and t-conorm is explained by the found two rules, and a new method is developed to compare alternatives with complete coverage of all possible $r$, which is demonstrated by two numerical examples and compared with two existing methods. Finally, Section 6 concludes this paper.

\section{Preliminaries}

In this section, we briefly review basic concepts of AIF, IVIF, HF, and DHF sets, and Hamacher t-norm and t-conorm.

\subsection{AIF and IVIF sets}

Atanassov [3] generalized the concept of fuzzy set [46], and defined the concept of 
AIF set as follows.

Definition 1 ([3]). Let $X=\left\{x_{1}, x_{2}, \ldots, x_{n}\right\}$ be a set, then an AIF set $\tilde{A}$ on $X$ is defined as

$$
\tilde{A}=\left\{\left\langle x, u_{\tilde{A}}(x), v_{\tilde{A}}(x)\right\rangle, x \in X\right\},
$$

where $u_{\tilde{A}}: X \rightarrow[0,1], v_{\tilde{A}}: X \rightarrow[0,1]$, and $0 \leq u_{\tilde{A}}(x)+v_{\tilde{A}}(x) \leq 1, \quad \forall x \in X$. For each $x \in X \quad, \quad u_{\tilde{A}}(x)$ and $v_{\tilde{A}}(x)$ represent the degrees of membership and non-membership of $x$ to $\tilde{A}$, respectively.

As an extension of AIF set, IVIF set was developed by Atanassov and Gargov [4]. Definition 2 ([4,24]). Let $X=\left\{x_{1}, x_{2}, \ldots, x_{n}\right\}$ be a universe of discourse. Then an IVIF set $\tilde{A}$ on $X$ is given by

$$
\tilde{A}=\left\{\left\langle x, \tilde{u}_{\tilde{A}}(x), \tilde{v}_{\tilde{A}}(x)\right\rangle, x \in X\right\},
$$

where $\tilde{u}_{\tilde{A}}(x)$ and $\tilde{v}_{\tilde{A}}(x)$ denote interval-valued membership and non-membership degrees of $x$ to $\tilde{A}$ such that $\tilde{u}_{\tilde{A}}(x) \subseteq[0,1], \tilde{v}_{\tilde{A}}(x) \subseteq[0,1]$, and $0 \leq \sup \left(\tilde{u}_{\tilde{A}}(x)\right)+$ $\sup \left(\tilde{v}_{\tilde{A}}(x)\right) \leq 1, \quad \forall x \in X$

For convenience, let $\tilde{u}_{\tilde{A}}\left(x_{i}\right)=[a, b], \quad \tilde{v}_{\tilde{A}}\left(x_{i}\right)=[c, d]$, then $\tilde{a}=([a, b],[c, d])$ is called an IVIF number [22]. The comparison between two IVIF numbers is defined as follows.

Definition 3 ([17]). Let $\tilde{a}=([a, b],[c, d])$ be an IVIF number, then the score function of $\tilde{a}$ is $S(\tilde{a})=\frac{a+b-c-d}{2}$, and the accuracy function of $\tilde{a}$ is $H(\tilde{a})=\frac{a+b+c+d}{2}$. For two IVIF numbers $\tilde{a}_{1}$ and $\tilde{a}_{2}$, if $S\left(\tilde{a}_{1}\right)>S\left(\tilde{a}_{2}\right)$, then $\tilde{a}_{1}>\tilde{a}_{2}$; if $S\left(\tilde{a}_{1}\right)=S\left(\tilde{a}_{2}\right), \quad \tilde{a}_{1}>\tilde{a}_{2}$ and $\tilde{a}_{1}=\tilde{a}_{2}$ can be deduced respectively from $H\left(\tilde{a}_{1}\right)>H\left(\tilde{a}_{2}\right)$ and $H\left(\tilde{a}_{1}\right)=H\left(\tilde{a}_{2}\right)$.

\subsection{HF and DHF sets}

Torra [32] first proposed the concept of HF set, which is defined as follows.

Definition 4 ([32]). Given a universe of discourse $X$, an HF set on $X$ is defined as 


$$
\tilde{A}=\left\{\left\langle x, h_{\tilde{A}}(x)\right\rangle \mid x \in X\right\}
$$

where $h_{\tilde{A}}(x)$ symbolizes possible membership degrees of $x$ to $\tilde{A}$, each of which is limited to $[0,1]$.

On the basis of HF set and AIF set, DHF set was developed by Zhu et al. [49].

Definition 5 ([49]). Given a universe of discourse $X$, a DHF set on $X$ is defined as

$$
\tilde{A}=\left\{\left\langle x, \tilde{h}_{\tilde{A}}(x), \tilde{g}_{\tilde{A}}(x)\right\rangle \mid x \in X\right\},
$$

where $\tilde{h}_{\tilde{A}}(x)$ and $\tilde{g}_{\tilde{A}}(x)$ denote possible membership and non-membership sets of $x$ to $\tilde{A}$ such that $\tilde{h}_{\tilde{A}}: X \rightarrow[0,1], \quad \tilde{g}_{\tilde{A}}: X \rightarrow[0,1]$, and $0 \leq \max \left\{\tilde{h}_{\tilde{A}}(x)\right\}+\max \left\{\tilde{g}_{\tilde{A}}(x)\right\} \leq 1$ for all $x \in X$.

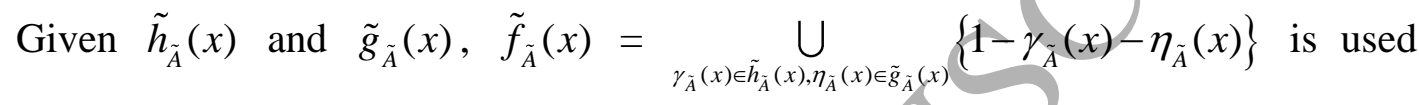
to symbolize a possible indeterminacy (uncertain) set of $x$ to $\tilde{A}$, where $\gamma_{\tilde{A}}(x) \in \tilde{h}_{\tilde{A}}(x) \quad$ and $\quad \eta_{\tilde{A}}(x) \in \tilde{g}_{\tilde{A}}(x) \quad$ represent possible membership and non-membership degrees of $x$ to $\tilde{A}$. For a specific $x, \tilde{a}=\{\tilde{h}, \tilde{g}\}$ is called a DHF element. Two DHF elements are compared by the following definition.

Definition 6 ([34,49]). Let $\tilde{a}=\{\tilde{h}, \tilde{g}\}$ be a DHF element, then the score function of $\tilde{a} \quad$ is $\quad S(\tilde{a})=\frac{\sum_{i=1}^{\delta(\tilde{h})} \gamma_{i}}{\delta(\tilde{h})}-\frac{\sum_{j=1}^{\delta(\tilde{g})} \eta_{j}}{\delta(\tilde{g})}$, and the accuracy function of $\tilde{a}$ is $H(\tilde{a})=\frac{\sum_{i=1}^{\delta(\tilde{h})} \gamma_{i}}{\delta(\tilde{h})}+\frac{\sum_{j=1}^{\delta(\tilde{g})} \eta_{j}}{\delta(\tilde{g})}$, where $\delta(\tilde{h})$ and $\delta(\tilde{g})$ symbolize the numbers of the elements in $\tilde{h}$ and $\tilde{g}$, respectively. For two DHF elements $\tilde{a}_{1}$ and $\tilde{a}_{2}$, if $S\left(\tilde{a}_{1}\right)>S\left(\tilde{a}_{2}\right)$, then $\tilde{a}_{1}>\tilde{a}_{2}$; if $S\left(\tilde{a}_{1}\right)=S\left(\tilde{a}_{2}\right), \tilde{a}_{1}>\tilde{a}_{2}$ and $\tilde{a}_{1}=\tilde{a}_{2}$ can be deduced respectively from $H\left(\tilde{a}_{1}\right)>H\left(\tilde{a}_{2}\right)$ and $H\left(\tilde{a}_{1}\right)=H\left(\tilde{a}_{2}\right)$.

\subsection{Hamacher t-norm and t-conorm}

T-norm and t-conorm are widely applied in fuzzy context to define the generalized intersection and union operations of fuzzy sets [11].

Definition 7 ([11]). A given function $T:[0,1] \times[0,1] \rightarrow[0,1]$ is called a t-norm when 
it satisfies the following four constraints:

(1) $T(1, x)=x$, for all $x$;

(2) $T(x, y)=T(y, x)$, for all $x$ and $y$;

(3) $T(x, T(y, z))=T(T(x, y), z)$, for all $x, y$, and $z$; and

(4) If $x \leq x_{1}$ and $y \leq y_{1}$, then $T(x, y) \leq T\left(x_{1}, y_{1}\right)$.

Definition 8 ([11]). A given function $S:[0,1] \times[0,1] \rightarrow[0,1]$ is called a t-conorm when it satisfies the following four constraints:

(1) $S(0, x)=x$, for all $x$;

(2) $S(x, y)=S(y, x)$, for all $x$ and $y$;

(3) $S(x, S(y, z))=S(S(x, y), z)$, for all $x, y$, and $z$;

(4) If $x \leq x_{1}$ and $y \leq y_{1}$, then $S(x, y) \leq S\left(x_{1}, y_{1}\right)$.

A continuous t-norm $T(x, y)$ such that $T(x, x)<x$ for all $x \in(0,1)$ is called an Archimedean t-norm. Similarly, an Archimedean t-conorm $S(x, y)$ satisfies that $S(x, x)$ $>x$ for all $x \in(0,1)$. Strict Archimedean t-norm and t-conorm are strictly increasing for all $x, y \in(0,1)$ [11]. A strict Archimedean t-norm $T(x, y)=p^{-1}(p(x)+p(y))$ can be created from a strictly decreasing function $p:[0,1] \rightarrow[0,+\infty]$ such that $p(1)=0$, whose dual function $q(x)=p(1-x)$ can be used to construct a strict Archimedean t-conorm $S(x, y)=q^{-1}(q(x)+q(y))$, as stated by Xia et al. [38].

Given a specific $p(x)$, i.e.,

$$
p(x)=\log \left(\frac{r+(1-r) x}{x}\right), r>0,
$$

it is clear that

$$
q(x)=p(1-x)=\log \left(\frac{r+(1-r) \cdot(1-x)}{1-x}\right) .
$$

Under this condition, strict Archimedean t-norm and t-conorm are called Hamacher t-norm $T_{r}(x, y)$ and t-conorm $S_{r}(x, y)$ [15], which are calculated by

$$
\begin{aligned}
& T_{r}(x, y)=\frac{x y}{r-(r-1) \cdot(x+y-x y)} \text { and } \\
& S_{r}(x, y)=\frac{x+y+(r-2) x y}{1+(r-1) x y}, r>0 .
\end{aligned}
$$

$T_{r}(x, y)$ and $S_{r}(x, y)$ are also called Hamacher product $\otimes$ and Hamacher sum $\oplus$ [15]. Specifically, $T_{r}(x, y)$ and $S_{r}(x, y)$ reduce to algebraic t-norm and t-conorm when $r$ $=1$; while they become Einstein t-norm and t-conorm when $r=2[6,13]$. 


\section{Propositions about Hamacher aggregation operators in MADM}

As analyzed in Introduction, Hamacher t-norm and t-conorm are applied in MADM problems with AIF, IVIF, HF, and DHF assessments to create aggregation operators so as to combine assessments of alternatives on each attribute. From presenting an analysis of numerical examples or real cases in existing studies concerning MADM with AIF, IVIF, HF, and DHF assessments, we find two common rules of Hamacher aggregation operators in the four types of MADM. As AIF and HF are special cases of IVIF and DHF, respectively, the following representative examples or cases regarding MADM with IVIF and DHF assessments will be examined to elicit the two rules.

\subsection{Analysis of Hamacher aggregation operators in MADM with IVIF assessments}

To evaluate the air quality of Guangzhou for the $16^{\text {th }}$ Asian Olympic Games, the air quality in Guangzhou for the Novembers of 2006, 2007, 2008,) and 2009 were evaluated to find out the trends in 2010. Liu [22] used Hamacher arithmetic hybrid weighted averaging operator and Hamacher geometric hybrid weighted averaging operator of IVIF information to combine IVIF assessments of each alternative on each attribute, from which evaluation scores of the air quality of Guangzhou for the Novembers of the four years were generated (see Table IV in [22] for details).

The evaluation results in [22] indicate the following: (1) evaluation scores of the air quality of Guangzhou for the Novembers of the four years decreased and increased with the increase of the parameter $r$ in the Hamacher arithmetic hybrid weighted averaging operator and Hamacher geometric hybrid weighted averaging operator of IVIF information; and (2) evaluation scores of the air quality of Guangzhou for the Novembers of the four years generated by the Hamacher arithmetic hybrid weighted averaging operator are always larger than those generated by the Hamacher geometric hybrid weighted averaging operator given different values of $r$.

\subsection{Analysis of Hamacher aggregation operators in MADM with DHF assessments}

To address MADM with DHF assessments, Ju et al. [19] developed a variety of DHF aggregation operators by combining Hamacher operations with averaging operator, weighted geometric operator and OWA operator. To handle a project evaluation problem, Hamacher arithmetic and geometric hybrid weighted averaging operators for DHF assessments under different $r$ are applied to aggregate the DHF assessments of four projects under consideration (see Table 2 in [19] for details).

The results generated in [19] reveal the following: (1) scores of the four projects decreased and increased with the increase of the parameter $r$ when the Hamacher arithmetic and geometric hybrid weighted averaging operators of DHF infromation are respectively applied; (2) scores of the four projects generated by the Hamacher 
arithmetic hybrid weighted averaging operator are always larger than those generated by the Hamacher geometric hybrid weighted averaging operator when $r$ is set as different values; and (3) the ranking order of the four projects have become different when $r$ in Hamacher geometric hybrid weighted averaging operator is changed from 0.5 to 1 . The third observation emphasizes the important influence of $r$ in Hamacher geometric hybrid weighted averaging operator on solutions to the project evaluation problem.

\subsection{Propositions}

Suppose that different arithmetic and geometric aggregation operators based on Hamacher operations are called Hamacher arithmetic and geometric aggregation operators, respectively. Two common rules can be extracted from the representative analysis of the movement of alternative scores in MADM with IVIF and DHF assessments with variation in the parameter $r$ in Hamacher arithmetic and geometric aggregation operators. They are formally presented below.

Proposition 1. Alternative scores decrease and increase with the increase of the parameter $r$ in Hamacher arithmetic and geometric aggregation operators respectively, when the operators are applied in MADM with IVIF and DHF assessments.

Proposition 2. Alternative scores generated by Hamacher arithmetic operator are always larger than those generated by Hamacher geometric operator given the same $r$ when the two operators are applied in MADM with IVIF and DHF assessments.

In the next section, the above two propositions will be theoretically proven in MADM with IVIF and DHF assessments.

\section{Theoretical proof of two propositions}

In this section, we prove the two propositions presented in Section 3.3 when Hamacher arithmetic and geometric aggregation operators are applied in MADM with IVIF and DHF assessments.

4.1 Proof of two propositions in MADM with IVIF assessments

\subsubsection{Description of MADM problems with IVIF assessments}

Suppose that a MADM problem has $m$ alternatives $A_{i}(i=1, \ldots, m)$ and $n$ attributes $C_{j}(j=1, \ldots, n)$. The relative weights of the $n$ attributes are represented by $\omega=\left(\omega_{1}\right.$,

$\left.\omega_{2}, \ldots, \omega_{n}\right)^{T}$ such that $0 \leq \omega_{j} \leq 1$ and $\sum_{i=1}^{n} \omega_{j}=1$, where the notation ' $T$ ' denotes 'transpose'. Let $\tilde{A}_{i j}=\left(\tilde{u}_{i j}, \tilde{v}_{i j}\right)=\left(\left[a_{i j}, b_{i j}\right],\left[c_{i j}, d_{i j}\right]\right)$ signify the IVIF assessment of 
alternative $A_{i}$ on attribute $C_{j}$. Then, an IVIF decision matrix for the problem can be profiled by

$$
\tilde{A}_{m \times n}=\left[\begin{array}{cccc}
<\tilde{u}_{11}, \tilde{v}_{11}> & <\tilde{u}_{12}, \tilde{v}_{12}> & \cdots & <\tilde{u}_{1 n}, \tilde{v}_{1 n}> \\
<\tilde{u}_{21}, \tilde{v}_{21}> & <\tilde{u}_{22}, \tilde{v}_{22}> & \cdots & <\tilde{u}_{2 n}, \tilde{v}_{2 n}> \\
\vdots & \vdots & \ddots & \vdots \\
<\tilde{u}_{m 1}, \tilde{v}_{m 1}> & <\tilde{u}_{m 2}, \tilde{v}_{m 2}> & \cdots & <\tilde{u}_{m n}, \tilde{v}_{m n}>
\end{array}\right],
$$

where each element represents an IVIF number.

\subsubsection{Monotonicity of alternative scores with respect to $r$ in Hamacher arithmetic and} geometric aggregation operators for MADM with IVIF assessments

For MADM with IVIF assessments, we address Proposition 1 to identify the monotonicity of alternative scores with respect to the parameter $r$ in Hamacher arithmetic and geometric aggregation operators.

To solve a MADM problem with IVIF assessments, the assessments of alternatives on each attribute are first combined using Hamacher aggregation operators to generate the aggregated assessments of alternatives. The aggregated assessments are used to calculate the scores of alternatives, and then to create a ranking order of alternatives. In most MADM methods, assessment $\tilde{A}_{i j}$ is only weighted by attribute weight $\omega_{j}(j=$ $1, \ldots, n)$ in the process of attribute combination, while the ordered position of $\tilde{A}_{i j}$ is usually omitted. Differently, both the attribute weight and the ordered position of $\tilde{A}_{i j}$ are involved in the Hamacher arithmetic and geometric hybrid weighted averaging operators developed by Liu [22]. It is clear that the aggregation of $\tilde{A}_{i j}$ only by $\omega_{j}$ in most MADM methods can be seen as a special case of the aggregation in Liu's method. Without loss of generality, in the following we focus on the two Hamacher aggregation operators created by Liu [22] to verify Proposition 1.

Definition 9 ([22]). Let the IVIF number $\tilde{A}_{i j}=\left(\tilde{u}_{i j}, \tilde{v}_{i j}\right)=\left(\left[a_{i j}, b_{i j}\right],\left[c_{i j}, d_{i j}\right]\right)(i=1$, $2, \ldots, m, j=1, \ldots, n)$ be the assessment of alternative $A_{i}$ on attribute $C_{j}$ for a MADM problem, $\omega=\left(\omega_{1}, \omega_{2}, \ldots, \omega_{n}\right)^{T}$ be the relative weights of the $n$ attributes, and $w_{i}=$ $\left(w_{i 1}, \ldots, w_{i n}\right)^{T}$ be the OWA operator weights with respect to $\tilde{A}_{i j}$. Then, the Hamacher arithmetic and geometric hybrid weighted averaging operators are defined below.

a) The aggregated assessment $\tilde{A}_{i}=\left(\tilde{u}_{i}, \tilde{v}_{i}\right)(i=1,2, \ldots, m)$ using the Hamacher arithmetic hybrid weighted averaging operator is defined as 


$$
\begin{aligned}
& \tilde{A}_{i}=w_{i l} \tilde{B}_{i \sigma(1)} \oplus \ldots \oplus w_{i n} \tilde{B}_{i \sigma(n)}= \\
& \left(\left[q^{-1}\left(\sum_{j=1}^{n} w_{i j} q\left(\dot{a}_{i \sigma(\mathrm{j})}\right)\right), q^{-1}\left(\sum_{j=1}^{n} w_{i j} q\left(\dot{b}_{i \sigma(j)}\right)\right)\right],\right. \\
& \left.\left[p^{-1}\left(\sum_{j=1}^{n} w_{i j} p\left(\dot{c}_{i \sigma(j)}\right)\right), p^{-1}\left(\sum_{j=1}^{n} w_{i j} p\left(\dot{d}_{i \sigma(j)}\right)\right)\right]\right)= \\
& \left(\left[\frac{\prod_{j=1}^{n}\left(1+(r-1) \dot{a}_{i \sigma(j)}\right)^{w_{i j}}-\prod_{j=1}^{n}\left(1-\dot{a}_{i \sigma(j)}\right)^{w_{i j}}}{\prod_{j=1}^{n}\left(1+(r-1) \dot{a}_{i \sigma(j)}\right)^{w_{i j}}+(r-1) \prod_{j=1}^{n}\left(1-\dot{a}_{i \sigma(j)}\right)^{w_{i j}}},\right.\right.
\end{aligned}
$$$$
\left.\frac{\prod_{j=1}^{n}\left(1+(r-1) \dot{b}_{i \sigma(j)}\right)^{w_{j j}}-\prod_{j=1}^{n}\left(1-\dot{b}_{i \sigma(j)}\right)^{w_{i j}}}{\prod_{j=1}^{n}\left(1+(r-1) \dot{b}_{i \sigma(j)}\right)^{w_{i j}}+(r-1) \prod_{j=1}^{n}\left(1-\dot{b}_{i \sigma(j)}\right)^{w_{i j}}}\right],
$$

$$
\left.\left[\begin{array}{c}
\frac{r \prod_{j=1}^{n}\left(\dot{c}_{i \sigma(j)}\right)^{w_{i j}}}{\prod_{j=1}^{n}\left(1+(r-1)\left(1-\dot{c}_{i \sigma(j)}\right)\right)^{w_{i j}}+(r-1) \prod_{j=1}^{n}\left(\dot{c}_{i \sigma(j)}\right)^{w_{i j}}} \\
\prod_{j=1}^{n}\left(1+(r-1)\left(1-\dot{d}_{i \sigma(j)}\right)\right)^{w_{i j}}+(r-1) \prod_{j=1}^{n}\left(\dot{d}_{i \sigma(j)}\right)^{w_{i j}} \\
\dot{w}_{i \sigma(j)}
\end{array}\right)^{w_{i j}}\right],
$$

where $\tilde{B}_{i \sigma(j)}$ stands for the $j$ th largest of $\tilde{B}_{i j}=n \omega_{i j} \tilde{A}_{i j}=\left(\left[\dot{a}_{i j}, \dot{b}_{i j}\right],\left[\dot{c}_{i j}, \dot{d}_{i j}\right]\right)$, and $(\sigma(1), \sigma(2), \ldots, \sigma(n))$ for a permutation of $(1,2, \ldots, n)$ such that $S\left(\tilde{B}_{i \sigma(j)}\right) \geq S\left(\tilde{B}_{i \sigma(j+1)}\right)$ $(j=1, \ldots, n-1)$.

b) The aggregated assessment $\tilde{A}_{i}=\left(\tilde{u}_{i}, \tilde{v}_{i}\right)(i=1,2, \ldots, m)$ using the Hamacher geometric hybrid weighted averaging operator is defined as

$$
\begin{aligned}
& \tilde{A}_{i}=\tilde{B}_{i \sigma(1)}^{w_{i 1}} \otimes \ldots \otimes \tilde{B}_{i \sigma(n)}^{w_{i n}}= \\
& \left(\left[p^{-1}\left(\sum_{j=1}^{n} w_{i j} p\left(\dot{a}_{i \sigma(j)}\right)\right), p^{-1}\left(\sum_{j=1}^{n} w_{i j} p\left(\dot{b}_{i \sigma(j)}\right)\right)\right],\right. \\
& \left.\left[q^{-1}\left(\sum_{j=1}^{n} w_{i j} q\left(\dot{c}_{i \sigma(j)}\right)\right), q^{-1}\left(\sum_{j=1}^{n} w_{i j} q\left(\dot{d}_{i \sigma(j)}\right)\right)\right]\right)=
\end{aligned}
$$




$$
\begin{aligned}
& \left(\left[\frac{r \prod_{j=1}^{n} \dot{a}_{i \sigma(j)}^{w_{i j}}}{\prod_{j=1}^{n}\left(1+(r-1)\left(1-\dot{a}_{i \sigma(j)}\right)\right)^{w_{i j}}+(r-1) \prod_{j=1}^{n}\left(1-\dot{a}_{i \sigma(j)}\right)^{w_{i j}}},\right.\right. \\
& \left.\frac{r \prod_{j=1}^{n} \dot{b}_{i \sigma(j)}^{w_{i j}}}{\prod_{j=1}^{n}\left(1+(r-1)\left(1-\dot{b}_{i \sigma(j)}\right)\right)^{w_{i j}}+(r-1) \prod_{j=1}^{n}\left(1-\dot{b}_{i \sigma(j)}\right)}\right], \\
& {\left[\frac{\prod_{j=1}^{n}\left(1+(r-1) \dot{c}_{i \sigma(j)}\right)^{w_{i j}}-\prod_{j=1}^{n}\left(1-\dot{c}_{i \sigma(j)}\right)^{w_{i j}}}{\prod_{j=1}^{n}\left(1+(r-1) \dot{c}_{i \sigma(j)}\right)^{w_{i j}}+(r-1) \prod_{j=1}^{n}\left(1-\dot{c}_{i \sigma(j)}\right)^{w_{i j}}},\right.} \\
& \left.\frac{\prod_{j=1}^{n}\left(1+(r-1) \dot{d}_{i \sigma(j)}\right)^{w_{i j}}-\prod_{j=1}^{n}\left(1-\dot{d}_{i \sigma(j)}\right)^{w_{i j}}}{\prod_{j=1}^{n}\left(1+(r-1) \dot{d}_{i \sigma(j)}\right)^{w_{i j}}+(r-1) \prod_{j=1}^{n}\left(1-\dot{d}_{i \sigma(j)}\right)^{w_{i j}}}\right],
\end{aligned}
$$

where $\tilde{B}_{i \sigma(j)}$ stands for the $j$ th largest of $\tilde{B}_{i j}=\tilde{A}_{i j}^{n \omega_{i j}}=\left(\left[\dot{a}_{i j}, \dot{b}_{i j}\right],\left[\dot{c}_{i j}, \dot{d}_{i j}\right]\right)$, and $(\sigma(1)$, $\sigma(2), \ldots, \sigma(n))$ for a permutation of $(1,2, \ldots, n)$ such that $S\left(\tilde{B}_{i \sigma(j)}\right) \geq S\left(\tilde{B}_{i \sigma(j+1)}\right) \quad(j=$ $1, \ldots, n-1)$.

The aggregated assessments of alternatives are then used to calculate the scores of alternatives in terms of Definition 3.

(1) Given the aggregated assessment $\tilde{A}_{i}=\left(\tilde{u}_{i}, \tilde{v}_{i}\right)=$

$\left(\left[q^{-1}\left(\sum_{j=1}^{n} w_{i j} q\left(\dot{a}_{i \sigma(j)}\right)\right), q^{-1}\left(\sum_{j=1}^{n} w_{i j} q\left(\dot{b}_{i \sigma(j)}\right)\right)\right]\right.$,

$\left[p^{-1}\left(\sum_{j=1}^{n} w_{i j} p\left(\dot{c}_{i \sigma(\mathrm{j})}\right)\right), p^{-1}\left(\sum_{j=1}^{n} w_{i j} p\left(\dot{d}_{i \sigma(\mathrm{j})}\right)\right)\right] \quad$ generated by the Hamacher arithmetic/hybrid weighted averaging operator, the score of $\tilde{A}_{i}$ denoted by $S^{a}\left(\tilde{A}_{i}\right)$ is calculated by

$$
\begin{aligned}
& S^{a}\left(\tilde{A}_{i}\right)=\frac{\left[q^{-1}\left(\sum_{j=1}^{n} w_{i j} q\left(\dot{a}_{i \sigma(\mathrm{j})}\right)\right)+q^{-1}\left(\sum_{j=1}^{n} w_{i j} q\left(\dot{b}_{i \sigma(\mathrm{j})}\right)\right)\right]}{2}- \\
& \frac{\left[p^{-1}\left(\sum_{j=1}^{n} w_{i j} p\left(\dot{c}_{i \sigma(\mathrm{j})}\right)\right)+p^{-1}\left(\sum_{j=1}^{n} w_{i j} p\left(\dot{d}_{i \sigma(\mathrm{j})}\right)\right)\right]}{2} .
\end{aligned}
$$

(2) Given the aggregated assessment $\tilde{A}_{i}=\left(\tilde{u}_{i}, \tilde{v}_{i}\right)=$ 
$\left(\left[p^{-1}\left(\sum_{j=1}^{n} w_{i j} p\left(\dot{a}_{i \sigma(\mathrm{j})}\right)\right), p^{-1}\left(\sum_{j=1}^{n} w_{i j} p\left(\dot{b}_{i \sigma(\mathrm{j})}\right)\right)\right]\right.$,

$\left.\left[q^{-1}\left(\sum_{j=1}^{n} w_{i j} q\left(\dot{c}_{i \sigma(\mathrm{j})}\right)\right), q^{-1}\left(\sum_{j=1}^{n} w_{i j} q\left(\dot{d}_{i \sigma(\mathrm{j})}\right)\right)\right]\right)$ generated by the Hamacher geometric hybrid weighted averaging operator, the score of $\tilde{A}_{i}$ denoted by $S^{g}\left(\tilde{A}_{i}\right)$ is calculated by

$$
\begin{aligned}
& S^{g}\left(\tilde{A}_{i}\right)=\frac{\left[p^{-1}\left(\sum_{j=1}^{n} w_{i j} p\left(\dot{a}_{i \sigma(\mathrm{j})}\right)\right)+p^{-1}\left(\sum_{j=1}^{n} w_{i j} p\left(\dot{b}_{i \sigma(\mathrm{j})}\right)\right)\right]}{2}- \\
& \frac{\left[q^{-1}\left(\sum_{j=1}^{n} w_{i j} q\left(\dot{c}_{i \sigma(\mathrm{j})}\right)\right)+q^{-1}\left(\sum_{j=1}^{n} w_{i j} q\left(\dot{d}_{i \sigma(\mathrm{j})}\right)\right)\right]}{2} .
\end{aligned}
$$

From Eqs. (12) and (13) we find that $S^{a}\left(\tilde{A}_{i}\right)$ (or $S^{g}\left(\tilde{A}_{i}\right)$ ) comprises two parts, which are

$$
\begin{aligned}
& \frac{\left[q^{-1}\left(\sum_{j=1}^{n} w_{i j} q\left(\dot{a}_{i \sigma(\mathrm{j})}\right)\right)+q^{-1}\left(\sum_{j=1}^{n} w_{i j} q\left(\dot{b}_{i \sigma(\mathrm{j})}\right)\right)\right]}{2} \\
& \left(\text { or } \frac{\left[p^{-1}\left(\sum_{j=1}^{n} w_{i j} p\left(\dot{a}_{i \sigma(\mathrm{j})}\right)\right)+p^{-1}\left(\sum_{j=1}^{n} w_{i j} p\left(\dot{b}_{i \sigma(\mathrm{j})}\right)\right)\right]}{2}\right) \text { and } \\
& \frac{\left[p^{-1}\left(\sum_{j=1}^{n} w_{i j} p\left(\dot{c}_{i \sigma(\mathrm{j})}\right)\right)+p^{-1}\left(\sum_{j=1}^{n} w_{i j} p\left(\dot{d}_{i \sigma(\mathrm{j})}\right)\right)\right]}{2} \\
& \text { (or } \left.\frac{\left[q^{-1}\left(\sum_{j=1}^{n} w_{i j} q\left(\dot{c}_{i \sigma(\mathrm{j})}\right)\right)+q^{-1}\left(\sum_{j=1}^{n} w_{i j} q\left(\dot{d}_{i \sigma(\mathrm{j})}\right)\right)\right]}{2}\right) .
\end{aligned}
$$

The definitions of two functions $p$ and $q$ in Eqs. (5) and (6) indicate that the two parts in $S^{a}\left(\tilde{A}_{i}\right)$ (or $\left.S^{g}\left(\tilde{A}_{i}\right)\right)$ are the functions with respect to the parameter $r$. In this context the verification of Proposition 1 is equivalently transformed into the discussion of the monotonicity of the functions $p$ and $q$ with respect to $r$. The relevant conclusions are drawn and shown in the following theorems.

Theorem 1. Suppose that $M(r)=q^{-1}\left(\sum_{j=1}^{n} w_{j} q\left(\mu_{j}\right)\right)$ is a function with the parameter $r$ where $0 \leq w_{j} \leq 1, \quad \sum_{j=1}^{n} w_{j}=1,0 \leq \mu_{j} \leq 1$, and $r \in(0,+\infty)$. Then, the function is monotonously decreasing with respect to $r$.

Theorem 2. Suppose that $N(r)=p^{-1}\left(\sum_{j=1}^{n} w_{j} p\left(\mu_{j}\right)\right)$ is a function with the parameter $r$ where $0 \leq w_{j} \leq 1, \sum_{j=1}^{n} w_{j}=1,0 \leq \mu_{j} \leq 1$, and $r \in(0,+\infty)$. Then, the 
function is monotonously increasing with respect to $r$.

The proofs of Theorems 1 and 2 are presented in Sections A.1 and A.2 of Appendix A of the supplementary material, respectively. From Eqs. (12) and (13) and Theorems 1 and 2 , we can draw the conclusion that $S^{a}\left(\tilde{A}_{i}\right)$ and $S^{g}\left(\tilde{A}_{i}\right)$ are the monotonously decreasing and increasing functions with respect to $r$, respectively. This reveals that Proposition 1 holds in the context of MADM with IVIF assessments when the Hamacher arithmetic and geometric hybrid weighted averaging operators are applied.

\subsubsection{Relationship between alternative scores derived from Hamacher arithmetic and} geometric aggregation operators for MADM with IVIF assessments

In the previous section, the monotonicity of $S^{a}\left(\tilde{A}_{i}\right)$ and $S^{g}\left(\tilde{A}_{i}\right)$ with respect to the parameter $r$ in Hamacher aggregation operators was theoretically proven. Based on this monotonicity, the relationship between $S^{a}\left(\tilde{A}_{i}\right)$ and $S^{g}\left(\tilde{A}_{i}\right)$ will be discussed and proven in the following. In other words, Proposition 2 in MADM with IVIF assessments will be verified. To facilitate the analysis of the relationship between $S^{a}\left(\tilde{A}_{i}\right)$ and $S^{g}\left(\tilde{A}_{i}\right)$, we firstly present two relevant lemmas.

Lemma 1 ([40]). Suppose that $x_{j}>0, \lambda_{j}>0(j=1, \ldots, n)$, and $\sum_{j=1}^{n} \lambda_{j}=1$, then we have

$$
\prod_{j=1}^{n} x_{j}^{\lambda_{j}} \leq \sum_{j=1}^{n} \lambda_{j} x_{j}
$$

with equality if and only if $x_{1}=x_{2}=\ldots=x_{n}$.

Lemma 2. The function $f(x)=\frac{x}{x+b}$ is monotonously increasing with respect to the parameter $x$, where $x>0$ and $b \geq 0$.

Lemma 2 is proven in Section A.3 of Appendix A. Based on the two lemmas, the relationship between $S^{a}\left(\tilde{A}_{i}\right)$ and $S^{g}\left(\tilde{A}_{i}\right)$ is presented in the following theorem.

Theorem 3. Suppose that $S^{a}\left(\tilde{A}_{i}\right)$ and $S^{g}\left(\tilde{A}_{i}\right)$ are the scores of the aggregated assessment $\tilde{A}_{i}$ generated by using the Hamacher arithmetic and geometric aggregation operators presented in Eqs. (12) and (13), respectively. Then, we have

$$
S^{a}\left(\tilde{A}_{i}\right)>S^{g}\left(\tilde{A}_{i}\right), \quad r \in(0,+\infty) .
$$

Theorem 3 is proven in Section A.4 of Appendix A with the use of Proposition 1, Lemma 1, and Lemma 2. This indicates that Proposition 2 holds in the context of 
MADM with IVIF assessments when the Hamacher arithmetic and geometric hybrid weighted averaging operators are applied.

\subsection{Proof of two propositions in MADM with DHF assessments}

\subsubsection{Description of MADM problems with DHF assessments}

For the same MADM problem introduced in Section 4.1.1, let $\tilde{A}_{i j}=\left\{h_{i j}, g_{i j}\right\}$ signify the DHF assessment of alternative $A_{i}$ on attribute $C_{j}$, where $0 \leq h_{i j}, g_{i j} \leq 1$ and $h_{i j}+g_{i j} \leq 1$. A DHF decision matrix for the problem is then given by

$$
\tilde{A}_{m \times n}=\left[\begin{array}{cccc}
<h_{11}, g_{11}> & <h_{12}, g_{12}> & \cdots & <h_{1 n}, g_{1 n}> \\
<h_{21}, g_{21}> & <h_{22}, g_{22}> & \cdots & <h_{2 n}, g_{2 n}> \\
\vdots & \vdots & \ddots & \vdots \\
<h_{m 1}, g_{m 1}> & <h_{m 2}, g_{m 2}> & \cdots & <h_{m n}, g_{m n}>
\end{array}\right] \text {. }
$$

In the following we verify Propositions 1 and 2 in MADM with DHF assessments.

4.2.2 Monotonicity of alternative scores with respect to $r$ in Hamacher arithmetic and geometric aggregation operators for MADM with DHF assessments

Similar to the situation in Section 4.1.2, to prove Proposition 1 in a general case of MADM with DHF assessments, the Hamacher arithmetic and geometric hybrid weighted averaging operators developed by $\mathrm{Ju}$ et al. [19] are used to combine assessments of alternatives on each attribute. The combination is defined as follows.

Definition 10 ([19]). Let the DHF element $\tilde{A}_{i j}=\left\{h_{i j}, g_{i j}\right\}=\left\{\bigcup_{\gamma_{i j} \in h_{i j}}\left\{\gamma_{i j}\right\}, \bigcup_{\eta_{i j} \in g_{i j}}\left\{\eta_{i j}\right\}\right\}(i=$ $1,2, \ldots, m, j=1, \ldots, n)$ be the assessment of alternative $A_{i}$ on attribute $C_{j}$ for a MADM problem, $\omega=\left(\omega_{1}, \omega_{2}, \ldots, \omega_{n}\right)^{T}$ be the relative weights of the $n$ attributes, and $w_{i}=\left(w_{i 1}, \ldots, w_{i n}\right)^{T}$ be the OWA operator weights with respect to $\tilde{A}_{i j}$. The Hamacher arithmetic and geometric hybrid weighted averaging operators are defined below.

a) The aggregated assessment of alternative $\tilde{A}_{i}=\left\{h_{i}, g_{i}\right\}(i=1,2, \ldots, m)$ using the Hamacher/arithmetic hybrid weighted averaging operator is defined as

$$
\begin{aligned}
& \tilde{A}_{i}=w_{i 1} \tilde{B}_{i \sigma(1)} \oplus \ldots \oplus w_{i n} \tilde{B}_{i \sigma(n)}= \\
& \left\{\bigcup_{\dot{\gamma}_{i \sigma(j)} \in \dot{h}_{i \sigma(j)}}\left\{q^{-1}\left(\sum_{j=1}^{n} w_{i j} q\left(\dot{\gamma}_{i \sigma(j)}\right)\right)\right\}, \bigcup_{\dot{i}_{i \sigma(j)} \in \dot{g}_{i \sigma(j)}}\left\{p^{-1}\left(\sum_{j=1}^{n} w_{i j} p\left(\dot{\eta}_{i \sigma(j)}\right)\right)\right\}\right\}= \\
& \left\{\bigcup_{\dot{\gamma}_{i \sigma(j)} \in \dot{h}_{i \sigma(j)}}\left\{\frac{\prod_{j=1}^{n}\left(1+(r-1) \dot{\gamma}_{i \sigma(j)}\right)^{w_{i j}}-\prod_{j=1}^{n}\left(1-\dot{\gamma}_{i \sigma(j)}\right)^{w_{i j}}}{\prod_{j=1}^{n}\left(1+(r-1) \dot{\gamma}_{i \sigma(j)}\right)^{w_{i j}}+(r-1) \prod_{j=1}^{n}\left(1-\dot{\gamma}_{i \sigma(j)}\right)^{w_{i j}}}\right\},\right.
\end{aligned}
$$




$$
\left.\bigcup_{\dot{\eta}_{i \sigma(j)} \in \dot{g}_{i \sigma(j)}}\left\{\frac{r \prod_{j=1}^{n} \dot{\eta}_{i \sigma(j)}^{w_{i j}}}{\prod_{j=1}^{n}\left(1+(r-1)\left(1-\dot{\eta}_{i \sigma(j)}\right)\right)^{w_{i j}}+(r-1) \prod_{j=1}^{n} \dot{\eta}_{i \sigma(j)}^{w_{i j}}}\right\}\right\},
$$

where $\tilde{B}_{i \sigma(j)}$ stands for the $j$ th largest of $\tilde{B}_{i j}=n \omega_{i j} \tilde{A}_{i j}=\left\{\dot{h}_{i j}, \dot{g}_{i j}\right\}=$ $\left\{\bigcup_{\gamma_{i j} \in h_{i j}}\left\{\dot{\gamma}_{i j}\right\}, \bigcup_{\eta_{i j} \in \dot{g}_{i j}}\left\{\dot{\eta}_{i j}\right\}\right\}$, and $(\sigma(1), \sigma(2), \ldots, \sigma(n))$ for a permutation of $(1,2, \ldots, n)$ such that $S\left(\tilde{B}_{i \sigma(j)}\right) \geq S\left(\tilde{B}_{i \sigma(j+1)}\right) \quad(j=1, \ldots, n-1)$.

b) The aggregated assessment of alternative $\tilde{A}_{i}=\left\{h_{i}, g_{i}\right\}(i=1,2, \ldots, m)$ using the Hamacher geometric hybrid weighted averaging operator is defined as

$$
\begin{aligned}
& \tilde{A}_{i}=\tilde{B}_{i \sigma(1)}^{w_{i 1}} \otimes \ldots \otimes \tilde{B}_{i \sigma(n)}^{w_{i n}}= \\
& \left\{\bigcup_{\dot{\gamma}_{i \sigma(j)} \in \dot{h}_{\sigma(j)}}\left\{p^{-1}\left(\sum_{j=1}^{n} w_{i j} p\left(\dot{\gamma}_{i \sigma(j)}\right)\right)\right\}, \bigcup_{\dot{\eta}_{i \sigma(j)} \in \dot{B}_{i \sigma(j)}}\left\{q^{-1}\left(\sum_{j=1}^{n} w_{i j} q\left(\dot{\eta}_{i \sigma(j)}\right)\right)\right\}\right\}= \\
& \left\{\bigcup_{\dot{\gamma}_{i \sigma(j)} \in \dot{h}_{i \sigma(j)}}\left\{\frac{r \prod_{j=1}^{n} \dot{\gamma}_{i(\sigma)}^{w_{i j}}}{\prod_{j=1}^{n}\left(1+(r-1)\left(1-\dot{\gamma}_{i \sigma(j)}\right)\right)^{w_{i j}}+(r-1) \prod_{j=1}^{n} \dot{\gamma}_{i \sigma(j)}^{w_{i j}}}\right\},\right. \\
& \left.\bigcup_{\left.\dot{\eta}_{i \sigma(j)}\right) \dot{g}_{i \sigma(j)}}\left\{\frac{\prod_{j=1}^{n}\left(1+(r-1) \dot{\eta}_{i \sigma(j)}\right)^{w_{i j}}-\prod_{j=1}^{n}\left(1-\dot{\eta}_{i \sigma(j)}\right)^{w_{i j}}}{\prod_{j=1}^{n}\left(1+(r-1) \dot{\eta}_{i \sigma(j)}\right)^{w_{i j}}+(r-1) \prod_{j=1}^{n}\left(1-\dot{\eta}_{i \sigma(j)}\right)^{w_{i j}}}\right\}\right\},
\end{aligned}
$$

where $\tilde{B}_{i \sigma(j)}$ stands for the jth largest of $\tilde{B}_{i j}=\tilde{A}_{i j}{ }^{n \omega_{i j}}=\left\{\dot{h}_{i j}, \dot{g}_{i j}\right\}=$ $\left\{\bigcup_{\gamma_{i j} \in h_{i j}}\left\{\dot{\gamma}_{i j}\right\}, \bigcup_{\eta_{i j} \in \dot{g}_{i j}}\left\{\dot{\eta}_{i j}\right\}\right\}$, and $(\sigma(1), \sigma(2), \ldots, \sigma(n))$ for a permutation of $(1,2, \ldots, n)$ such that $S\left(\tilde{B}_{i \sigma(j)}\right) \geq S\left(\tilde{B}_{i \sigma(j+1)}\right) \quad(j=1, \ldots, n-1)$.

Similar to the situation in Section 4.1.2, based on the aggregated assessments of alternative $A_{i}$ in Definition 10, the corresponding scores denoted by $S^{a}\left(\tilde{A}_{i}\right)$ and $S^{g}\left(\tilde{A}_{i}\right)$, can be calculated using Definition 6, as shown below.

(1) Given the aggregated assessment $\tilde{A}_{i}=\left\{h_{i}, g_{i}\right\}=$

$$
\left\{\bigcup_{\dot{\gamma}_{i \sigma(j)} \in \dot{h}_{\sigma(j)}}\left\{q^{-1}\left(\sum_{j=1}^{n} w_{i j} q\left(\dot{\gamma}_{i \sigma(j)}\right)\right)\right\}, \bigcup_{\dot{\eta}_{i \sigma(j)} \in \dot{g}_{\sigma \sigma(j)}}\left\{p^{-1}\left(\sum_{j=1}^{n} w_{i j} p\left(\dot{\eta}_{i \sigma(j)}\right)\right)\right\}\right\} \text { generated }
$$

by the Hamacher arithmetic hybrid weighted averaging operator, the score of $\tilde{A}_{i}$ denoted by $S^{a}\left(\tilde{A}_{i}\right)$ is calculated by 


$$
S^{a}\left(\tilde{A}_{i}\right)=\frac{\sum\left[q^{-1}\left(\sum_{j=1}^{n} w_{i j} q\left(\dot{\gamma}_{i \sigma(j)}\right)\right)\right]}{\delta\left(h_{i}\right)}-\frac{\sum\left[p^{-1}\left(\sum_{j=1}^{n} w_{i j} p\left(\dot{\eta}_{i \sigma(j)}\right)\right)\right]}{\delta\left(g_{i}\right)}
$$

where $\delta\left(h_{i}\right)$ and $\delta\left(g_{i}\right)$ symbolize the numbers of the elements in $h_{i}$ and $g_{i}$, respectively.

(2) Given the aggregated assessment $\tilde{A}_{i}=\left\{h_{i}, g_{i}\right\}=$

$$
\left\{\bigcup_{\dot{\gamma}_{i \sigma(j)} \in \dot{h}_{i \sigma(j)}}\left\{p^{-1}\left(\sum_{j=1}^{n} w_{i j} p\left(\dot{\gamma}_{i \sigma(j)}\right)\right)\right\}, \bigcup_{\dot{\eta}_{i \sigma(j)} \in \dot{g}_{i \sigma(j)}}\left\{q^{-1}\left(\sum_{j=1}^{n} w_{i j} q\left(\dot{\eta}_{i \sigma(j)}\right)\right)\right\}\right\} \text {, generated }
$$

by the Hamacher geometric hybrid weighted averaging operator, the score of $\tilde{A}_{i}$ denoted by $S^{g}\left(\tilde{A}_{i}\right)$ is calculated by

$$
S^{g}\left(\tilde{A}_{i}\right)=\frac{\sum\left[p^{-1}\left(\sum_{j=1}^{n} w_{i j} p\left(\dot{\gamma}_{i \sigma(j)}\right)\right)\right]}{\delta\left(h_{i}\right)}-\frac{\sum\left[q^{-1}\left(\sum_{j=1}^{n} w_{i j} q\left(\dot{\eta}_{i \sigma(j)}\right)\right)\right]}{\delta\left(g_{i}\right)},
$$

where $\delta\left(h_{i}\right)$ and $\delta\left(g_{i}\right)$ symbolize the numbers of the elements in $h_{i}$ and $g_{i}$, respectively.

Eqs. (19) and (20) indicate that $S^{a}\left(\tilde{A}_{i}\right)\left(\right.$ or $\left.S^{g}\left(\tilde{A}_{i}\right)\right)$ includes two parts, which are

$$
\begin{aligned}
& \left.\frac{\sum\left[q^{-1}\left(\sum_{j=1}^{n} w_{i j} q\left(\dot{\gamma}_{i \sigma(j)}\right)\right)\right]}{\delta\left(h_{i}\right)} \text { (or } \frac{\sum\left[p^{-1}\left(\sum_{j=1}^{n} w_{i j} p\left(\dot{\gamma}_{i \sigma(j)}\right)\right)\right]}{\delta\left(h_{i}\right)}\right) \text { and } \\
& \frac{\sum\left[p^{-1}\left(\sum_{j=1}^{n} w_{i j} p\left(\dot{\eta}_{i \sigma(j)}\right)\right)\right]}{\delta\left(g_{i}\right)}\left(\text { or } \frac{\sum\left\{q^{-1}\left(\sum_{j=1}^{n} w_{i j} q\left(\dot{\eta}_{i \sigma(j)}\right)\right)\right]}{\delta\left(g_{i}\right)}\right) .
\end{aligned}
$$

Similar to the situation in Section 4.1.2, $S^{a}\left(\tilde{A}_{i}\right)$ and $S^{g}\left(\tilde{A}_{i}\right)$ are the functions with respect to the parameter $r$ in Hamacher aggregation operators. Specifically, $S^{a}\left(\tilde{A}_{i}\right)$ and $S^{g}\left(\tilde{A}_{i}\right)$ in Eqs. (19) and (20) include the linear combination of multiple functions $q^{-1}$ and of $p^{-1}$. To facilitate analysis of the monotonicity of $S^{a}\left(\tilde{A}_{i}\right)$ and $S^{g}\left(\tilde{A}_{i}\right)$ with consideration of this specificity, we present the following lemma.

Lemma 3. Suppose that there is a set $H=\left\{h_{i}\right\}(i=1, \ldots, n)$ such that $0 \leq h_{i} \leq 1$, then the function $F\left(h_{1}, \ldots, h_{n}\right)=\frac{1}{n} \sum_{i=1}^{n} h_{i}$ is monotonously increasing with respect to $h_{i}$ $\left(\forall h_{i} \in H\right)$.

Lemma 3 clearly holds and thus its proof is omitted. Owing to Lemma 3 and 
Theorems 1 and 2, it can be concluded that $S^{a}\left(\tilde{A}_{i}\right)$ and $S^{g}\left(\tilde{A}_{i}\right)$ are the monotonously decreasing and increasing functions with respect to $r$, respectively. This indicates that Proposition 1 holds in the context of MADM with DHF assessments when the Hamacher arithmetic and geometric hybrid weighted averaging operators are applied.

\subsubsection{Relationship between alternative scores derived from Hamacher arithmetic and} geometric aggregation operators for MADM with DHF assessments

Similarly to Section 4.1.3, we discuss the relationship between $S^{a}\left(\tilde{A}_{i}\right)$ and $S^{g}\left(\tilde{A}_{i}\right)$ in MADM with DHF assessments. This relationship is presented in the following theorem.

Theorem 4. Suppose that $S^{a}\left(\tilde{A}_{i}\right)$ and $S^{g}\left(\tilde{A}_{i}\right)$ are the scores of the aggregated assessment $\tilde{A}_{i}$ generated by using the Hamacher arithmetic and geometric aggregation operators, as presented in Eqs. (19) and (20), respectively. Then, we have

$$
S^{a}\left(\tilde{A}_{i}\right)>S^{g}\left(\tilde{A}_{i}\right), \quad r \in(0,+\infty) .
$$

Theorem 4 is proven in Section A.5 of Appendix A with the use of Proposition 1 and Lemmas 2 and 3. This shows that Proposition 2 holds in the context of MADM with DHF assessments when the Hamacher arithmetic and geometric hybrid weighted averaging operators are applied.

It should be noted that two functions $p$ and $q$ in Eqs. (5) and (6) are not the unique choices for the Hamacher t-norm and t-conorm. For other functions $p$ and $q$ such that $q^{-1}$ and $q$ are monotonously increasing (or decreasing) and $p^{-1}$ and $p$ are monotonously increasing (or decreasing), Propositions 1 and 2 still hold in MADM with IVIF or DHF assessments.

\section{A method for ranking alternatives in MADM problems with DHF assessments under the two propositions}

The analysis in Section 4 indicates that the parameter $r$ in Hamacher aggregation operators has a significant effect on the aggregated assessments of alternatives, and further on the solution to a MADM problem. With the use of Propositions 1 and 2, in the following, we will discuss the meaning of the parameter $r$ and develop a new method to compare alternatives when handling MADM problems with DHF assessments. Two numerical examples are solved by the proposed method to demonstrate its applicability and validity. They also help to conduct a comparison between the developed method and two existing methods to highlight the consistency and validity of the developed method. 


\subsection{Meaning of the parameter $r$ in Hamacher aggregation operators}

From previous studies $[16,19,22,26,30,39,48]$, we can conclude that there are two main issues concerning the parameter $r$ in Hamacher aggregation operators when handling MADM problems: (1) the meaning of $r$ is not clear; and (2) the determination of $r$ is arbitrary and subjective.

Regarding the first issue, in existing studies many researchers have associated $r$ with the preference or risk attitude of a decision maker (e.g., $[30,38])$. However, the reason for such an association is not clear. Some studies explain that $r$ can be characterized by the utility of a decision maker (e.g., [16,22,39,48]). However, no convinced explanation exists which is commonly accepted by researchers for lack of theoretical proof.

Regarding the second issue, numerical examples and real cases in existing studies $[19,22,48]$ have found that the precise value of $r$ is usually specified when Hamacher aggregation operators are applied to solve MADM problems. However, such specification of $r$ is generally ill-founded and arbitrary, and mainly depends on the subjectivity of the decision maker. It may become even more difficult when a variety of types of information are involved in determining $r$.

In this paper, Proposition 1 and its theoretical proof indicate that $r$ can be reasonably associated with the risk attitude, in terms of the optimism and pessimism of a decision maker. To elaborate, a decision maker is risk-seeking when he or she prefers small $r$, while the decision maker is risk-averse if he or she prefers large $r$ when the Hamacher arithmetic aggregation operator is applied in MADM with IVIF or DHF assessments. The opposite conclusion can be drawn when the Hamacher geometric aggregation operator is applied in MADM with IVIF or DHF assessments. In the former situation, small $r$ indicates a large alternative score, while it indicates a small alternative score in the latter. Although the meaning of $r$ is clear, determining the precise value of $r$ from the interval $(0,+\infty)$ remains a difficult task for the decision maker, especially when the decision maker knows various types of information concerning $r$. In response to this difficulty, in the next section we propose a new method to compare alternatives in MADM which considers all possible values of $r$ instead of a specific value.

5.2 Method for comparing alternatives in MADM problems with DHF assessments under the two propositions

As analyzed above, although the parameter $r$ in Hamacher aggregation operators can reflect the optimistic or pessimistic attitude of a decision maker according to Proposition 1, it may be difficult to determine the precise value of $r$ from the interval $(0,+\infty)$. This is especially the case when the decision maker knows various types of 
relevant information. To avoid the negative influence of arbitrary or subjective values of $r$ upon decision results in MADM with DHF assessments, we propose a method for comparing alternatives with a full coverage of all possible values of $r$. The method in the context of MADM with IVIF assessments can be similarly developed, which is omitted here to save space.

Given that the Hamacher arithmetic aggregation operator is applied in MADM with DHF assessments, the score of alternative $A_{i}$ decreases monotonously with the increase of $r$ according to Proposition 1, which is plotted in Figure 1. In this condition, the mean score index of alternative $A_{i}$ with consideration of all possible values of $r$ such that $r \in(0,+\infty)$ is designed to compare alternatives, which is presented below.

Definition 11. Let $S^{a}\left(\tilde{A}_{i}\right)$ and $S^{g}\left(\tilde{A}_{i}\right)$ be the scores of alternative $A_{i}$ from Hamacher arithmetic and geometric aggregation operators. Then, the arithmetic and geometric mean score indexes of alternative $A_{i}$ on $r \in(0,+\infty)$ are defined as

$$
\begin{gathered}
A M I_{i}=\lim _{r_{0} \rightarrow+\infty} \frac{\int_{0^{+}}^{r_{0}} S^{a}\left(\tilde{A}_{i}\right) d r}{\left|r_{0}-0\right|}=\lim _{r_{0} \rightarrow+\infty} \frac{\int_{0^{+}}^{r_{0}} S^{a}\left(\tilde{A}_{i}\right) d r}{r_{0}}, \text { and } \\
G M I_{i}=\lim _{r_{0} \rightarrow+\infty} \frac{\int_{0^{+}}^{r_{0}} S^{g}\left(\tilde{A}_{i}\right) d r}{\left|r_{0}-0\right|}=\lim _{r_{0} \rightarrow+\infty} \frac{\int_{0^{+}}^{r_{0}} S^{g}\left(\tilde{A}_{i}\right) d r}{r_{0}} .
\end{gathered}
$$

Here, the elongated " $\int$ " represents integral.

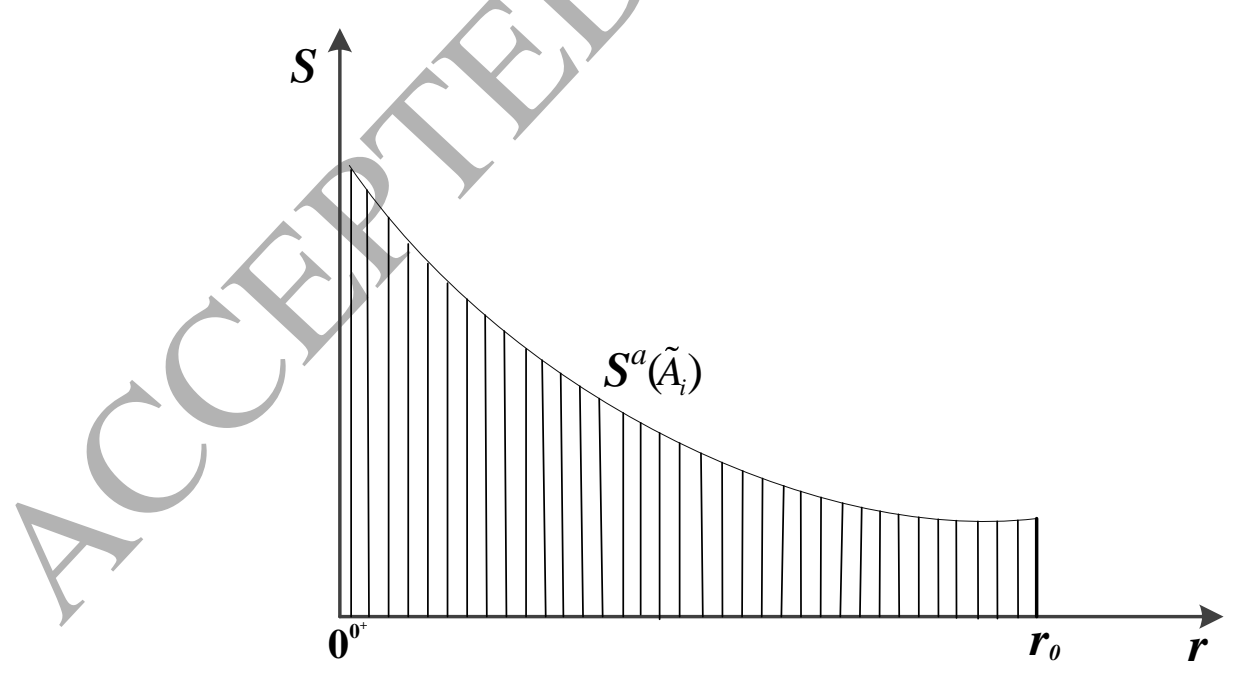

Figure. 1. Score movement of alternative $A_{i}$ with variation in $r$

To facilitate the calculation of $A M I_{i}$, it should first be identified whether the integral $\int_{0^{+}}^{r_{0}} S^{a}\left(\tilde{A}_{i}\right) d r$ with $r_{0} \rightarrow+\infty$ is divergent or convergent. As $\lim _{r_{0} \rightarrow+\infty} \int_{0^{+}}^{r_{0}} S^{a}\left(\tilde{A}_{i}\right) d r=$ 
$\int_{0^{+}}^{+\infty} S^{a}\left(\tilde{A}_{i}\right) d r$, the problem is transformed into deciding whether $\int_{0^{+}}^{+\infty} S^{a}\left(\tilde{A}_{i}\right) d r$ is divergent or convergent. To address this problem, the Cauchy criterion for convergence $[1,27]$ is introduced, as shown below.

Theorem 5 (Cauchy criterion for convergence) ([1,27]). Let $\varphi(x)$ and $\phi(x)$ be two functions with nonnegative terms, and $\int_{a}^{+\infty} \phi(x) d x$ and $\int_{a}^{+\infty} \varphi(x) d x$ be the integrals of $\phi(x)$ and $\varphi(x)$ on $[a,+\infty)$, respectively. Suppose that $0 \leq \varphi(x) \leq$ $k \phi(x)$ for all $x$ in $[a,+\infty)$, where $a$ is a real number and $k>0$. Then,

(i) If $\int_{a}^{+\infty} \phi(x) d x$ converges, $\int_{a}^{+\infty} \varphi(x) d x$ also converges; and

(ii) If $\int_{a}^{+\infty} \varphi(x) d x$ diverges, $\int_{a}^{+\infty} \phi(x) d x$ also diverges.

Theorem 5 reveals that when the lower bound of $S^{a}\left(\tilde{A}_{i}\right)$ exists on $r \in(0,+\infty)$, the divergence of $\int_{0^{+}}^{+\infty} S^{a}\left(\tilde{A}_{i}\right) d r$ can be identified by the Cauchy criterion for convergence. To carry out the identification, the lower bound of $S^{a}\left(\tilde{A}_{i}\right)$ on $r \in(0,+\infty)$ is determined in the following proposition.

Proposition 3. Let $\tilde{A}_{i}=\left\{h_{i}, g_{i}\right\}$ be the arithmetic aggregated assessment of alternative $A_{i}$, where $\left\{h_{i}, g_{i}\right\}=$

$$
\begin{aligned}
& \left\{\bigcup_{\dot{\gamma}_{i \sigma(j)} \in h_{i}}\left\{\frac{\prod_{j=1}^{n}\left(1+(r-1) \dot{\gamma}_{i \sigma(j)}\right)^{w_{i j}}-\prod_{j=1}^{n}\left(1-\dot{\gamma}_{i \sigma(j)}\right)^{w_{i j}}}{\prod_{j=1}^{n}\left(1+(r-1) \dot{\gamma}_{i \sigma(j)}\right)^{w_{i j}}+(r-1) \prod_{j=1}^{n}\left(1-\dot{\gamma}_{i \sigma(j)}\right)^{w_{i j}}}\right\},\right. \\
& \left.\bigcup_{\dot{\eta}_{i \sigma(j)} \in g_{i}}\left\{\frac{r \prod_{j=1}^{n} \dot{\eta}_{i \sigma(j)}^{w_{i j}}}{\prod_{j=1}^{n}\left(1+(r-1)\left(1-\dot{\eta}_{i \sigma(j)}\right)\right)^{w_{i j}}+(r-1) \prod_{j=1}^{n} \dot{\eta}_{i \sigma(j)}^{w_{i j}}}\right\}\right\}, \text { and the score function }
\end{aligned}
$$

of $\tilde{A}_{i}$ be $S^{a}\left(\tilde{A}_{i}\right)$ with $r \in(0,+\infty)$. Then, the lower bound of the score function $S^{a}\left(\tilde{A}_{i}\right)$ with $r \in(0,+\infty)$ is $S\left(\tilde{\tilde{A}}_{i}\right)$, where $\tilde{\tilde{A}}_{i}=\{h, g\}=\lim _{r \rightarrow+\infty} \tilde{A}_{i}=$

$$
\left\{\bigcup_{\dot{\gamma}_{i \sigma(j)} \in h_{i}}\left\{\frac{\prod_{j=1}^{n}\left(\dot{\gamma}_{i \sigma(j)}\right)^{w_{i j}}}{\prod_{j=1}^{n}\left(\dot{\gamma}_{i \sigma(j)}\right)^{w_{i j}}+\prod_{j=1}^{n}\left(1-\dot{\gamma}_{i \sigma(j)}\right)^{w_{i j}}}\right\},\right.
$$




$$
\bigcup_{\dot{\eta}_{i \sigma(j)} \in g_{i}}\left\{\frac{\prod_{j=1}^{n}\left(\dot{\eta}_{i \sigma(j)}\right)^{w_{i j}}}{\prod_{j=1}^{n}\left(\dot{\eta}_{i \sigma(j)}\right)^{w_{i j}}+\prod_{j=1}^{n}\left(1-\dot{\eta}_{i \sigma(j)}\right)^{w_{i j}}}\right\} \text {, i.e. } S^{a}\left(\tilde{A}_{i}\right)>S\left(\tilde{\tilde{A}}_{i}\right) \text {. }
$$

The conclusions of Proposition 3 can be directly inferred from Proposition 1, the proof of Theorem 4 and Definition 6, so the proof is omitted here. With the use of Theorem 5 and Proposition 3, it is possible to identify whether or not $\int_{0^{+}}^{+\infty} S^{a}\left(\tilde{A}_{i}\right) d r$ is divergent.

Theorem 6. Let $\tilde{A}_{i}=\left\{h_{i}, g_{i}\right\}$ be the arithmetic aggregated assessment of alternative $A_{i}$, where $\left\{h_{i}, g_{i}\right\}=\left\{\bigcup_{\dot{\gamma}_{i \sigma(j)} \in h_{i}}\left\{\frac{\prod_{j=1}^{n}\left(1+(r-1) \dot{\gamma}_{i \sigma(j)}\right)^{w_{i j}}-\prod_{j=1}^{n}\left(1-\dot{\gamma}_{i \sigma(j)}\right)^{w_{i j}}}{\prod_{j=1}^{n}\left(1+(r-1) \dot{\gamma}_{i \sigma(j)}\right)^{w_{i j}}+(r-1) \prod_{j=1}^{n}\left(1-\dot{\gamma}_{i \sigma(j)}\right)^{w_{i j}}}\right\}\right.$, $\left.\bigcup_{\dot{\eta}_{i \sigma(j)} \in g_{i}}\left\{\frac{r \prod_{j=1}^{n} \dot{\eta}_{i \sigma(j)}^{w_{i j}}}{\prod_{j=1}^{n}\left(1+(r-1)\left(1-\dot{\eta}_{i \sigma(j)}\right)\right)^{w_{i j}}+(r-1) \prod_{j=1}^{n} \widehat{\dot{\eta}_{i \sigma(j)}^{w_{i j}}}}\right\}\right\}$ and the score function of $\tilde{A}_{i}$ be $S^{a}\left(\tilde{A}_{i}\right)$ with $r \in(0,+\infty)$. Then, $\int_{0^{+}}^{+\infty} S^{a}\left(\tilde{A}_{i}\right) d r$ is divergent.

Theorem 6 is proved in Section A.6 of Appendix A. Similar to the discussions about the divergence of $\int_{0^{+}}^{+\infty} S^{a}\left(\tilde{A}_{i}\right) d r$, the divergence of $\int_{0^{+}}^{+\infty} S^{g}\left(\tilde{A}_{i}\right) d r$ can also be identified in order to calculate $G M I_{i}$. For this purpose, the lower bound of $S^{g}\left(\tilde{A}_{i}\right)$ with $r \in(0,+\infty)$ is first determined.

Proposition 4. Let $\tilde{A}_{i}=\left\{h_{i}, g_{i}\right\}$ be the geometric aggregated assessment of alternative $A_{i}$, where $\left\{h_{i}, g_{i}\right\}=$

$$
\begin{aligned}
& \left\{\bigcup_{\dot{\gamma}_{i \sigma(j)} \in \dot{h}_{i \sigma(j)}}\left\{\frac{r \prod_{j=1}^{n} \dot{\gamma}_{i \sigma(j)}^{w_{i j}}}{\prod_{j=1}^{n}\left(1+(r-1)\left(1-\dot{\gamma}_{i \sigma(j)}\right)\right)^{w_{i j}}+(r-1) \prod_{j=1}^{n} \dot{\gamma}_{i \sigma(j)}^{w_{i j}}}\right\},\right. \\
& \left.\bigcup_{\dot{\eta}_{i \sigma(j)} \in \dot{g}_{i \sigma(j)}}\left\{\frac{\prod_{j=1}^{n}\left(1+(r-1) \dot{\eta}_{i \sigma(j)}\right)^{w_{i j}}-\prod_{j=1}^{n}\left(1-\dot{\eta}_{i \sigma(j)}\right)^{w_{i j}}}{\prod_{j=1}^{n}\left(1+(r-1) \dot{\eta}_{i \sigma(j)}\right)^{w_{i j}}+(r-1) \prod_{j=1}^{n}\left(1-\dot{\eta}_{i \sigma(j)}\right)^{w_{i j}}}\right\}\right\}, \text { and the score }
\end{aligned}
$$

function of $\tilde{A}_{i}$ be $S^{g}\left(\tilde{A}_{i}\right)$ with $r \in(0,+\infty)$. Then, the lower bound of the score function $S^{g}\left(\tilde{A}_{i}\right)$ with $r \in(0,+\infty)$ is $S\left(\tilde{\tilde{A}}_{i}\right)$, where $\tilde{\tilde{A}}_{i}=\{h, g\}=\lim _{r \rightarrow 0^{+}} \tilde{A}_{i}=$ 


$$
\left\{\bigcup_{\dot{\gamma}_{i \sigma(j)} \in h_{i}}\left\{\frac{1}{1+\sum_{j=1}^{n} \frac{w_{i j}\left(1-\dot{\gamma}_{i \sigma(j)}\right)}{\dot{\gamma}_{i \sigma(j)}}}\right\}, \bigcup_{\dot{\eta}_{i \sigma(j)} \in g_{i}}\left\{\frac{\sum_{j=1}^{n} \frac{w_{i j} \cdot \dot{\eta}_{i \sigma(j)}}{\left(1-\dot{\eta}_{i \sigma(j)}\right)}}{1+\sum_{j=1}^{n} \frac{w_{i j} \cdot \dot{\eta}_{i \sigma(j)}}{\left(1-\dot{\eta}_{i \sigma(j)}\right)}}\right\}\right\} \text {, i.e. } S^{g}\left(\tilde{A}_{i}\right)>
$$

$S\left(\tilde{\tilde{A}}_{i}\right)$.

Proposition 4 is proven in Section A.7 of Appendix A. With the use of Theorem 5 and Proposition 4, whether $\int_{0^{+}}^{+\infty} S^{g}\left(\tilde{A}_{i}\right) d r$ is divergent can be identified.

Theorem 7. Let $\tilde{A}_{i}=\left\{h_{i}, g_{i}\right\}$ be the geometric aggregated assessment of alternative $A_{i}$, where $\left\{h_{i}, g_{i}\right\}=\left\{\bigcup_{\dot{\gamma}_{i \sigma(j)} \in \dot{h}_{i \sigma(j)}}\left\{\frac{r \prod_{j=1}^{n} \dot{\gamma}_{i \sigma(j)}^{w_{i j}}}{\prod_{j=1}^{n}\left(1+(r-1)\left(1-\dot{\gamma}_{i \sigma(j)}\right)\right)^{w_{j i}}+(r-1) \prod_{j=1}^{n} \dot{\gamma}_{i \sigma(j)}^{w_{j i}}}\right\}\right.$, $\left.\bigcup_{\dot{\eta}_{i \sigma(j)} \in \dot{B}_{i \sigma(j)}}\left\{\frac{\left.\prod_{j=1}^{n}\left(1+(r-1) \dot{\eta}_{i \sigma(j)}\right)^{w_{i j}}-\prod_{j=1}^{n}\left(1-\dot{\eta}_{i \sigma(j)}\right)^{w_{j j}}\right)}{\prod_{j=1}^{n}\left(1+(r-1) \dot{\eta}_{i \sigma(j)}\right)^{w_{j i}}+(r-1) \prod_{j=1}^{n}\left(1-\dot{\eta}_{i \sigma(j)}\right)^{w_{j j}}}\right\}\right\}$, and the score function of $\tilde{A}_{i}$ be $S^{g}\left(\tilde{A}_{i}\right)$ with $r \in(0,+\infty)$. Then, $\int_{0^{+}}^{+\infty} S^{g}\left(\tilde{A}_{i}\right) d r$ is divergent.

Theorem 7 is proved in Section A. 8 of Appendix A. As $\int_{0^{+}}^{+\infty} S^{a}\left(\tilde{A}_{i}\right) d r$ and $\int_{0^{+}}^{+\infty} S^{g}\left(\tilde{A}_{i}\right) d r$ are divergent, L'Hôpital's rule (also called Bernoulli's rule) [1] can be used in Eqs. (22) and (23) to calculate the values of $A M I_{i}$ and $G M I_{i}$.

Theorem 8. Let $\tilde{A}_{i}=\left\{h_{i}, g_{i}\right\}$ be the arithmetic aggregated assessment of alternative $A_{i}$, and $A M r_{i}$ be the arithmetic mean score index of alternative $A_{i}$ defined in Definition 11. Then we have $A M I_{i}=S\left(\tilde{\tilde{A}}_{i}\right)$ when $r \rightarrow+\infty$, where $\tilde{\tilde{A}}_{i}=\{h, g\}$ $=\lim _{r \rightarrow+\infty} \tilde{A}_{j}=$

$$
\left\{\bigcup_{\dot{i}_{i \sigma(j)} \in h_{i}}\left\{\frac{\prod_{j=1}^{n} \dot{\gamma}_{i \sigma(j)}^{w_{i j}}}{\prod_{j=1}^{n} \dot{\gamma}_{i \sigma(j)}^{w_{i j}}+\prod_{j=1}^{n}\left(1-\dot{\gamma}_{i \sigma(j)}\right)^{w_{i j}}}\right\}, \bigcup_{\dot{\eta}_{i \sigma(j)} \in g_{i}}\left\{\frac{\prod_{j=1}^{n} \dot{\eta}_{i \sigma(j)}^{w_{i j}}}{\prod_{j=1}^{n} \dot{\eta}_{i \sigma(j)}^{w_{j j}}+\prod_{j=1}^{n}\left(1-\dot{\eta}_{i \sigma(j)}\right)^{w_{i j}}}\right\}\right\} .
$$

Theorem 9. Let $\tilde{A}_{i}=\left\{h_{i}, g_{i}\right\}$ be the geometric aggregated assessment of alternative $A_{i}$, and $G M I_{i}$ be the geometric mean score index of alternative $A_{i}$. Then we have 


$$
\begin{gathered}
G M I_{i}=S\left(\tilde{\tilde{A}}_{i}\right) \text { when } r \rightarrow+\infty, \text { where } \tilde{\tilde{A}}_{i}=\{h, g\}=\lim _{r \rightarrow+\infty} \tilde{A}_{i}= \\
\left\{\bigcup_{\dot{\gamma}_{i \sigma(j)} \in h_{i}}\left\{\frac{\prod_{j=1}^{n} \dot{\gamma}_{i \sigma(\mathrm{j})}^{w_{i j}}}{\prod_{j=1}^{n} \dot{\gamma}_{i \sigma(\mathrm{j})}^{w_{i j}}+\prod_{j=1}^{n}\left(1-\dot{\gamma}_{i \sigma(\mathrm{j})}\right)^{w_{i j}}}\right\}, \bigcup_{\dot{\eta}_{i \sigma(j)} \in g_{i}}\left\{\frac{\prod_{j=1}^{n} \dot{\eta}_{i \sigma(\mathrm{j})}^{w_{i j}}}{\prod_{j=1}^{n} \dot{\eta}_{i \sigma(\mathrm{j})}^{w_{i j}}+\prod_{j=1}^{n}\left(1-\dot{\eta}_{i \sigma(\mathrm{j})}\right)^{w_{i j}}}\right\}\right\} .
\end{gathered}
$$

Theorems 8 and 9 are proven respectively in Sections A.9 and A.10 of Appendix A, respectively, with the use of Theorems 6 and 7 and L'Hôpital's rule. It is interesting to find from Theorems 8 and 9 that $A M I_{i}=G M I_{i}$. That is, we can use $A M I_{i}$ to compare alternatives regardless of whether or not Hamacher arithmetic or geometric aggregation operators are applied in MADM with DHF assessments.

Definition 12. Suppose that $A M I_{i}(i=1,2)$ represents the arithmetic mean score index of alternative $A_{i}$ with $r \in(0,+\infty)$. When $A M I_{1}>A M I_{2}$, alternative $A_{y}$ is said to be superior to alternative $A_{2}$.

\subsection{Numerical examples}

In this section, two numerical examples are solved by the method proposed in Section 5.2 to demonstrate its applicability and validity. The first example is originated from a real application where a large Chinese company in the iron and steel industry decides to invest abroad. The second one is also a practical example taken from the paper of Ju et al. [19].

Example 1. The iron and steel industry is one of the fundamental industries which contribute to China's economy. This industry is closely related to upstream and downstream industries and is driven by the requirements of consumption greatly. It also significantly influences the development of economy and society in China. As an important and essential resource for producing steel, iron directly restricts the development of the iron and steel industry. Unfortunately, iron resources in China are relatively limited and their quality is below the international average. To participate in international competition, large Chinese companies in the iron and steel industry must seek high-quality iron resources globally.

Taking a large domestic company in the iron and steel industry as an example, we investigate how to assist the company to invest abroad effectively and reasonably. The general manager of this company must decide which of the following five countries to invest in and source iron ores from: Australia $\left(A_{1}\right)$, India $\left(A_{2}\right)$, Brazil $\left(A_{3}\right)$, Canada $\left(A_{4}\right)$, or Russia $\left(A_{5}\right)$. Seven attributes are identified based on the annual surveys released by the Fraser Institute of Canada [18], and their weights are specified as $\omega=(0.35,0.1$, $0.05,0.1,0.05,0.15,0.2)$. The seven attributes are described in Table 1.

\section{Table 1}


Description of the seven attributes.

\begin{tabular}{ll}
\hline Attribute & Description \\
\hline$C_{1}$ & Quality and quantity of iron ore resources \\
$C_{2}$ & Situation of the legal system, taxation regime, and trade barriers \\
$C_{3}$ & Competition from other overseas investment in iron resources \\
$C_{4}$ & Uncertainty about environmental regulations and availability of skilled \\
& labor \\
$C_{5}$ & Infrastructure concerning overseas investments \\
$C_{6}$ & Condition of socioeconomic agreements/community development \\
$C_{7}$ & Political stability and security level \\
\hline
\end{tabular}

To make the decision, several academics, including two co-authors of this paper and four experts from the Chinese Academy of Engineering (CAE) and the Ministry of Land and Resources (MLR) were invited to independently and anonymously evaluate the five countries on each attribute. The evaluation and the preference of the decision makers are then combined to construct a DHF decision matrix $\tilde{A}_{5 \times 7}=\left(\left\{h_{i j}\right.\right.$, $\left.\left.g_{i j}\right\}\right)_{5 \times 7}$, which is shown in Section B.1 of Appendix B. For example, Australia $\left(A_{1}\right)$ is assessed on attribute $C_{2}$ as $\{\{0.6,0.7\},\{0.1,0.2\}\}$, which indicates that the degree to which alternative $A_{1}$ satisfies attribute $C_{2}$ may be 0.6 or 0.7 , and the degree to which alternative $A_{1}$ does not satisfy attribute $C_{2}$ may be 0.1 or 0.2 .

Assume that the OWA operator weight vector is specified as $w=(1 / 7,1 / 7,1 / 7,1 / 7$, $1 / 7,1 / 7,1 / 7)$. When the Hamacher arithmetic hybrid weighted averaging operator in Definition 10 is applied, the aggregated assessment of alternative $A_{i}$ is given by

$$
\begin{aligned}
& \tilde{A}_{i}=\left\{\bigcup_{\dot{\gamma}_{i \sigma(j)} \in \dot{h}_{i \sigma(j)}}\left\{\frac{\prod_{j \leq 1}^{7}\left(1+(r-1) \dot{\gamma}_{i \sigma(j)}\right)^{w_{i j}}-\prod_{j=1}^{7}\left(1-\dot{\gamma}_{i \sigma(j)}\right)^{w_{i j}}}{\prod_{j=1}^{7}\left(1+(r-1) \dot{\gamma}_{i \sigma(j)}\right)^{w_{i j}}+(r-1) \prod_{j=1}^{7}\left(1-\dot{\gamma}_{i \sigma(j)}\right)^{w_{i j}}}\right\},\right. \\
& \bigcup_{\dot{\eta}_{j \sigma \phi(j)} \in \dot{g}_{i \sigma(j)}}\left\{\frac{r \prod_{j=1}^{7} \dot{\eta}_{i \sigma(j)}^{w_{i j}}}{\prod_{j=1}^{7}\left(1+(r-1)\left(1-\dot{\eta}_{i \sigma(j)}\right)\right)^{w_{i j}}+(r-1) \prod_{j=1}^{7} \dot{\eta}_{i \sigma(j)}^{w_{i j}}}\right\} \text {. }
\end{aligned}
$$

The arithmetic mean score $A M I_{i}(i=1, \ldots, 5)$ is subsequently calculated, using Theorem 8, as $(0.2886,0.1680,0.2180,0.2404,0.0801)$. Using Theorem 9, the geometric mean score $G_{M I}(i=1, \ldots, 5)$ is also computed to be $(0.2886,0.1680,0.2180,0.2404$, 0.0801). The same value of $A M I_{i}$ and $G M I_{i}$ generates a common ranking order of the five countries, i.e. $A_{1} \succ A_{4} \succ A_{3} \succ A_{2} \succ A_{5}$, where the notation ' $\succ$ ' represents 'superior to'. Consequently, the optimal choice for investment is $A_{1}$ (Australia).

Example 2. Reconsider the example investigated by Ju et al. [19], in which the board director of a company intends to plan the development of large projects in the 
following five years. Four projects denoted by $A_{i}(i=1,2,3,4)$ are evaluated on four attributes including financial perspective $\left(C_{1}\right)$, customer satisfaction $\left(C_{2}\right)$, international business process perspective $\left(C_{3}\right)$, and learning and growth perspective $\left(C_{4}\right)$. The relative weights of the four attributes and the OWA operator weight vector are specified as $\omega=(0.2,0.15,0.35,0.3)$ and $w=(0.25,0.25,0.25,0.25)$, respectively. The DHF decision matrix $\tilde{A}_{4 \times 4}=\left(\left\{h_{i j}, g_{i j}\right\}\right)_{4 \times 4}$ of the example is presented in Section B.2 of Appendix B.

When the Hamacher arithmetic hybrid weighted averaging operator in Definition 10 is applied, the aggregated assessment of alternative $A_{i}$ is given by

$$
\begin{aligned}
& \tilde{A}_{i}=\left\{\bigcup_{\dot{\gamma}_{i \sigma(j)} \in \dot{h}_{i \sigma(j)}}\left\{\frac{\prod_{j=1}^{4}\left(1+(r-1) \dot{\gamma}_{i \sigma(j)}\right){ }^{w_{i j}}-\prod_{j=1}^{4}\left(1-\dot{\gamma}_{i \sigma(j)}\right)^{w_{i j}}}{\prod_{j=1}^{4}\left(1+(r-1) \dot{\gamma}_{i \sigma(j)}\right)^{w_{i j}}+(r-1) \prod_{j=1}^{4}\left(1-\dot{\gamma}_{i \sigma(j)}\right)^{w_{i j}}}\right\},\right. \\
& \bigcup_{\dot{\eta}_{i \sigma(j)} \in \dot{g}_{i \sigma(j)}}\left\{\frac{r \prod_{j=1}^{4} \dot{\eta}_{i \sigma(j)}^{w_{i j}}}{\left.\prod_{j=1}^{4}\left(1+(r-1)\left(1-\dot{\eta}_{i \sigma(j)}\right)\right)^{w_{i j}}+(r-1) \prod_{j=1}^{4} \dot{\eta}_{i \sigma(j)}^{w_{i j}}\right\}}\right\} .
\end{aligned}
$$

The arithmetic and geometric mean scores $A M I_{i}$ and $G M I_{i}(i=1, \ldots, 4)$ can then be calculated, using Theorems 8 and 9, both of which are equal to $(0.0333,0.1173$, $0.1219,0.009)$. A ranking order of the four projects is then generated as $A_{3} \succ A_{2} \succ A_{1} \succ A_{4}$. Consequently, the most desirable project is $A_{3}$. A comparison between the developed method and the methods of Ju et al. [19] and Ye [45] is made based on the above two examples in the next section.

\subsection{Comparative analysis}

In the following, the proposed method is compared with two representative methods developed by $\mathrm{Ju}$ et al. [19] and Ye [45] to highlight its consistency and validity.

The key ideas of the two existing methods are briefly described as follows. In the method of Ju et al. [19], the Hamacher arithmetic or geometric aggregation operator is utilized to combine the assessments on each attribute for each alternative. The values of the parameter $r$ in Hamacher aggregation operators are given first by decision makers. After the aggregated assessments of alternatives are obtained, a ranking order of alternatives is generated using Definition 6. In Ye's method [45], a virtual ideal solution is used as a reference to compare alternatives. An alternative with performance close to the ideal solution is preferred. Its closeness is measured by the correlation coefficient between the performance of the alternative and that of the ideal solution. The ranking order of alternatives is generated by using their closeness to the 
ideal solution.

In order to compare the proposed method with those of Ju et al. and $\mathrm{Ye}$, the two numerical examples in Section 5.3 were solved by using the latter two methods. The results of applying all three methods to the first example are presented in Table B.3 of Section B.3 in Appendix B. From Table B.3, we can see that the proposed method and the method of Ju et al. (using the Hamacher arithmetic aggregation operator) generate the same ranking order of the five countries: $A_{1} \succ A_{4} \succ A_{3} \succ A_{2} \succ A_{5}$. It should be noted that in the method of Ju et al., the ranking orders generated by Hamacher arithmetic aggregation operators differ from those generated by Hamacher geometric aggregation operators when $r \in\{0.5,1,2\}$. More importantly, the ranking orders generated by Hamacher arithmetic and geometric aggregation operators change with different given values of $r$. However, the ranking orders generated by the Hamacher arithmetic and geometric hybrid weighted averaging operators are always the same in the method proposed here. By using Ye's method, we can obtain the weighted correlation coefficients between the alternatives and the ideal solution as $(0.8914$, $0.8191,0.8096,0.8672,0.8043)$. From Table B.3, we can see that the ranking order generated by Ye's method is $A_{1} \succ A_{4} \succ A_{2} \succ A_{3} \succ A_{5}$, where the rankings of $A_{2}$ and $A_{3}$ differ from those generated by the proposed method, but the best choice is still $A_{1}$.

The results generated by the three methods for the second numerical example are presented in Table B.4 of Section B.3. It is shown that the ranking orders of the four projects derived from the Hamacher arithmetic and geometric hybrid weighted averaging operators in the proposed method are always the same: $A_{3} \succ A_{2} \succ A_{1} \succ A_{4}$. On the contrary, the ranking orders generated by the Hamacher arithmetic and geometric aggregation operators in the method of Ju et al. are different. When a method generates inconsistent outcomes, a decision maker may have difficulty in choosing which one to follow. Moreover, the value of $r$ influences decision results, and so to determine the value is another burden on a decision maker. In the proposed method, however, these two problems are handled without any burden on a decision maker. As shown in Table B.4, the ranking order of the four projects generated by Ye's method is $A_{3} \succ A_{2} \succ A_{4} \succ A_{1}$, in which the rankings of $A_{1}$ and $A_{4}$ differ from those generated by the proposed method, but the best alternative is still $A_{3}$.

In summary, the proposed method is able to generate more consistent decision results independent of the choice of Hamacher arithmetic and geometric aggregation operators and the value of $r$, compared with Ju et al.' method. This also indicates that the proposed method is more efficient than that of $\mathrm{Ju}$ et al. from the perspective of decision makers' involvement in a decision process. The best choices generated by the proposed method are always the same as those generated by the methods of $\mathrm{Ye}$ and $\mathrm{Ju}$ et al. The above comparative analysis highlights the consistency and validity of the 
method proposed in this paper.

\section{Conclusions}

When finding solutions to fuzzy MADM problems, Hamacher t-norms and t-conorms with a parameter $r$ are commonly used to combine various styles of fuzzy assessments on each attribute, such as AIF, IVIF, HF, and DHF assessments. Although different Hamacher aggregation operators have been developed to combine various styles of fuzzy assessments, the existing operators can generally be divided into two categories: Hamacher arithmetic and Hamacher geometric aggregation operators.

Since AIF and HF assessments are special cases of IVIF and DHF assessments, from numerical examples and real cases with IVIF and DHF assessments in the literature we present two propositions: (1) alternative scores decrease and increase with the increase of $r$ in Hamacher arithmetic and geometric aggregation operators; and (2) alternative scores generated by Hamacher arithmetic operators are always larger than those generated by Hamacher geometric operators given the same $r$. These two propositions are theoretically proven in the context of MADM with IVIF and DHF assessments. They are further applied to illustrate the meaning of $r$ and to develop a method for comparing alternatives in MADM with DHF assessments with full coverage of all possible values of $r$. Two numerical examples are solved by the proposed method to demonstrate its applicability and validity, and a comparative analysis is conducted to illustrate the consistency and validity of the method proposed in this paper.

The main contributions of this paper include the following: (1) two propositions concerning Hamacher arithmetic and geometric aggregation operators in the context of fuzzy MADM are presented following a thorough review and analysis of the literature on MADM with IVIF and DHF information; (2) these two propositions are proven theoretically in the context of MADM with IVIF and DHF assessments; (3) the meaning of the parameter $r$ in Hamacher arithmetic and geometric aggregation operators is explained using Proposition 1 and its theoretical proof; and (4) a new method is proposed for ranking alternatives in MADM problems with DHF information under the two propositions.

In recent decades, many developments have been achieved concerning the combination of multiple pieces of uncertain information in decision making. Despite this, some related scientific problems still require focused research. For example: 1) the selection of the type of aggregation functions [7];2) the studies about the admissible orders in terms of aggregation functions for various fuzzy sets [24]; and 3) the choice of score functions of different fuzzy assessments, as studied in [12] and [31]. In addition, granular computing [25] is a flexible and feasible tool for decision 
makers to address the challenges of characterizing their preferences in an uncertain context. Recently, a number of achievements [2,23,28,29,36,42,44] have been made in this area which contribute to further studies of uncertain decision making. All these areas are worth exploring in future research to solve uncertain MADM problems.

\section{Acknowledgements}

This research was supported by the National Natural Science Foundation of China (Grant Nos. 71622003, 71571060, 71521001, 71201043, 71131002, 71303073, and 71501054), and partially supported by the European Commission under Grant No. EC- GPF-314836. We are also grateful to the anonymous reviewers for their constructive comments.

\section{References}

[1] H. Anton, I. Bivens, S. Davis, Calculus, Beijing: Higher Education Press, 2008.

[2] B. Apolloni, S. Bassis, J. Rota, G.L. Galliani, M. Gioia, L. Ferrari, A neurofuzzy algorithm for learning from complex granules, Granular Computing 1 (2016). DOI 10.1007/s41066-016-0018-1.

[3] K.T. Atanassov, Intuitionistic fuzzy sets, Fuzzy Sets and Systems 20 (1) (1986) $87-96$.

[4] K.T. Atanassov, G. Gargov, Interval valued intuitionistic fuzzy sets, Fuzzy Sets and Systems 31 (3) (1989) 343-349.

[5] G. Beliakov, H. Bustince, S. James, T. Calvo, J. Fernandez, Aggregation for Atanassov's intuitionistic and interval valued fuzzy sets: the median operator, IEEE Transactions on Fuzzy Systems 20 (3) (2012) 487-498.

[6] G. Beliakov, A. Pradera, T. Calvo, Conjunctive and Disjunctive Functions, Heidelberg, Germany; Springer, 2007, pp. 123-196.

[7] H. Bustince, E. Barrenechea, T. Calvo, S. James, G. Beliakov, Consensus in multi-expert decision making problems using penalty functions defined over a Cartesian product of lattices, Information Fusion 17 (2014) 56-64.

[8] S.M. Chen, S.H. Cheng, T.C. Lan, Multicriteria decision making based on the TOPSIS method and similarity measures between intuitionistic fuzzy values, Information Sciences 367-368 (2016) 279-295.

[9] S.M. Chen, S.H. Cheng, W.H. Tsai, Multiple attribute group decision making based on interval-valued intuitionistic fuzzy aggregation operators and transformation techniques of interval-valued intuitionistic fuzzy values, Information Sciences 367-368 (2016) 418-442.

[10]J.F. Chu, X.W. Liu, Y.M. Wang, K.S. Chin, A group decision making model considering both the additive consistency and group consensus of intuitionistic fuzzy preference relations, Computers \& Industrial Engineering 101 (2016) 
227-242.

[11]G. Deschrijver, E.E. Kerre, A generalization of operators on intuitionistic fuzzy sets using triangular norms and conorms, Notes on IFS 8 (1) (2002) 19-27.

[12]H. Garg, A new generalized improved score function of interval-valued intuitionistic fuzzy sets and applications in expert systems, Applied Soft Computing 38 (2016) 988-999.

[13]H. Garg, Generalized intuitionistic fuzzy interactive geometric interaction operators using Einstein t-norm and t-conorm and their application to decision making, Computers \& Industrial Engineering 101 (2016) 53-69.

[14]P. Gupta, M. K. Mehlawat, N. Grover, Intuitionistic fuzzy multi-attribute group decision-making with an application to plant location selection based on a new extended VIKOR method, Information Sciences 370-371 (2016) 184-203.

[15]H. Hamachar, Uber logische verknunpfungenn unssharfer Aussagen undderen Zugenhorige Bewertungsfunktione, Progress in Cybernatics and systems research 3 (1978) 276-288.

[16]J.Y. Huang, Intuitionistic fuzzy Hamacher aggregation operators and their application to multiple attribute decision making, Journal of Intelligent \& Fuzzy Systems 27 (1) (2014) 505-513.

[17]W.L. Hung, J.W. Wu, Correlation of intuitionistic fuzzy sets by centroid method, Information Sciences 144 (1) (2002) 219-225.

[18]T. Jackson, K.P. Green, Fraser Institute Annual Survey of Mining Companies: 2015[EB/OL].https://www.fraserinstitute.org/studies/annual-survey-of-mining-co mpanies-2015.

[19] Y.B. Ju, W.K. Zhang, S.H. Yang, Some dual hesitant fuzzy Hamacher aggregation operators and their applications to multiple attribute decision making, Journal of Intelligent \& Fuzzy Systems 27 (5) (2014) 2481-2495.

[20]H.C. Liao, Z.S. Xu, Extended hesitant fuzzy hybrid weighted aggregation operators and their application in decision making, Soft Computing 19 (9) (2015) 2551-2564.

[21]H.C. Liao, Z.S. Xu, Intuitionistic fuzzy hybrid weighted aggregation operators, International Journal of Intelligent Systems 29 (11) (2014) 971-993.

[22]P.D. Liu, Some Hamacher aggregation operators based on the interval-valued intuitionistic fuzzy numbers and their application to group decision making, IEEE Transactions on Fuzzy systems 22 (1) (2014) 83-97.

[23]J.M. Mendel, A comparison of three approaches for estimating (synthesizing) an interval type-2 fuzzy set model of a linguistic term for computing with words, Granular Computing 1 (1) (2016) 59-69.

[24]L.D. Miguel, H. Bustince, J. Fernandez, E. Induráin, A. Kolesárová, R. Mesiar, 
Construction of admissible linear orders for interval-valued Atanassov intuitionistic fuzzy sets with an application to decision making, Information Fusion 27 (2016) 189-197.

[25]W. Pedrycz, S.M. Chen, Granular Computing and Decision-Making: Interactive and Iterative Approaches, Heidelberg, Germany: Springer, 2015.

[26]S. Roychowdhury, B.H. Wang, On generalized Hamacher families of triangular operators, International journal of approximate reasoning 19 (3) (1998) 419-439.

[27]B. Schweizer, A. Sklar, Statistical metric spaces, Pacific Journal of Mathematics 10 (1) (1960) 313-334.

[28]A. Skowron, A. Jankowski, Interactive granular computing, Granular Computing 1 (2) (2016) 95-113.

[29] M.L. Song, Y.B. Wang, A study of granular computing in the agenda of growth of artificial neural networks, Granular Computing 1 ) (2016). DOI 10.1007/s41066-016-0020-7.

[30]C.Q. Tan, W.T. Yi, X.H. Chen, Hesitant fuzzy Hamacher aggregation operators for multicriteria decision making, Applied Soft Computing 26 (2015) 325-349.

[31]N. Thillaigovindan, S.A. Shanthi, J.V. Naidu, A better score function for multiple criteria decision making in fuzzy environment with criteria choice under risk, Expert Systems with Applications 59 (2016) 78-85.

[32] V. Torra, Hesitant fuzzy sets, International Journal of Intelligent Systems 25 (6) (2010) 529-539.

[33]S.P. Wan, G.L. Xu, J.Y. Dong, A novel method for group decision making with interval-valued Atanassov intuitionistic fuzzy preference relations, Information Sciences 372 (2016) 53-71.

[34]L. Wang, Q.G. Shen, L. Zhu, Dual hesitant fuzzy power aggregation operators based on Archimedean t-conorm and t-norm and their application to multiple attribute group decision making, Applied Soft Computing 38 (2016) 23-50.

[35]H.J. Wang, X.F. Zhao, G.W. Wei, Dual hesitant fuzzy aggregation operators in multiple attribute decision making, Journal of Intelligent \& Fuzzy Systems 26 (5) (2014) 2281-2290.

[36]G. Wilke, E. Portmann, Granular computing as a basis of human-data interaction: a cognitive cities use case, Granular Computing 1 (3) (2016) 181-197.

[37]M.M. Xia, Z.S. Xu, Hesitant fuzzy information aggregation in decision making, International journal of approximate reasoning 52 (3) (2011) 395-407.

[38]M.M. Xia, Z.S. Xu, B. Zhu, Some issues on intuitionistic fuzzy aggregation operators based on Archimedean t-conorm and t-norm, Knowledge-Based Systems 31 (2012) 78-88.

[39]S. Xiao, Induced interval-valued intuitionistic fuzzy Hamacher ordered weighted 
geometric operator and their application to multiple attribute decision making, Journal of Intelligent \& Fuzzy Systems 27 (1) (2014) 527-534.

[40]Z.S. Xu, On consistency of the weighted geometric mean complex judgement matrix in AHP, European Journal of Operational Research 126 (3) (2000) 683-687.

[41]Z.S. Xu, H.C. Liao, A survey of approaches to decision making with intuitionistic fuzzy preference relations, Knowledge-based systems 80 (2015) 131-142.

[42]Z.S. $\mathrm{Xu}, \mathrm{H}$. Wang, Managing multi-granularity linguistic information in qualitative group decision making: An overview, Granular Computing 1(1) (2016) 21-35.

[43]Z.S. Xu, R.R. Yager, Some geometric aggregation operators based on intuitionistic fuzzy sets, International journal of general systems 35 (4) (2006) 417-433.

[44] Y.Y. Yao, A triarchic theory of granular computing, Granular Computing 1 (2) (2016) 145-157.

[45]J. Ye, Correlation coefficient of dual hesitant fuzzy sets and its application to multiple attribute decision making, Applied Mathematical Modelling 38 (2) (2014) 659-666.

[46]L.A. Zadeh, Fuzzy sets, Information and control 8 (3) (1965) 338-353.

[47]X.F. Zhao, G.W. Wei, Some intuitionistic fuzzy Einstein hybrid aggregation operators and their application to multiple attribute decision making, Knowledge-Based Systems 37 (2013) 472-479.

[48]L.Y. Zhou, X.F. Zhao, G.W. Wei, Hesitant fuzzy Hamacher aggregation operators and their application to multiple attribute decision making, Journal of Intelligent \& Fuzzy Systems 26 (6) (2014) 2689-2699.

[49]B. Zhu, Z.S. Xu, M.M. Xia, Dual hesitant fuzzy sets, Journal of Applied Mathematics (2012). DOI 10.1155/2012/879629. 